\title{
Compact Star-forming Galaxies as Old Starbursts Becoming Quiescent
}

Gómez-Guijarro, C.; Magdis, G. E.; Valentino, F.; Toft, S.; Man, A. W. S.; Ivison, R. J.; Tisani, K.; van der Vlugt, D.; Stockmann, M.; Martin-Alvarez, S.

Total number of authors:

11

Published in:

Astrophysical Journal Supplement Series

Link to article, DOI:

$10.3847 / 1538-4357 / a b 418 b$

Publication date:

2019

Document Version

Publisher's PDF, also known as Version of record

Link back to DTU Orbit

Citation (APA):

Gómez-Guijarro, C., Magdis, G. E., Valentino, F., Toft, S., Man, A. W. S., Ivison, R. J., Tisani, K., van der Vlugt, D., Stockmann, M., Martin-Alvarez, S., \& Brammer, G. (2019). Compact Star-forming Galaxies as Old Starbursts Becoming Quiescent. Astrophysical Journal Supplement Series, 886(2), [88]. https://doi.org/10.3847/1538$4357 / a b 418 b$

\section{General rights}

Copyright and moral rights for the publications made accessible in the public portal are retained by the authors and/or other copyright owners and it is a condition of accessing publications that users recognise and abide by the legal requirements associated with these rights.

- Users may download and print one copy of any publication from the public portal for the purpose of private study or research.

- You may not further distribute the material or use it for any profit-making activity or commercial gain

- You may freely distribute the URL identifying the publication in the public portal 


\title{
Compact Star-forming Galaxies as Old Starbursts Becoming Quiescent
}

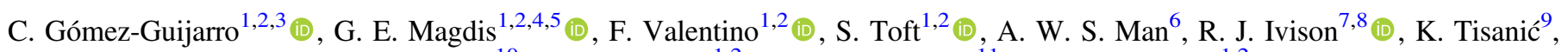 \\ D. van der Vlugt ${ }^{10}$, M. Stockmann ${ }^{1,2}$, S. Martin-Alvarez ${ }^{11}$, and G. Brammer ${ }^{1,2}$ (iD \\ ${ }^{1}$ Cosmic Dawn Center (DAWN), Denmark; carlos.gomez@nbi.ku.dk \\ ${ }^{2}$ Niels Bohr Institute, University of Copenhagen, Lyngbyvej 2, DK-2100 Copenhagen, Denmark \\ ${ }^{3}$ Laboratoire AIM-Paris-Saclay, CEA/DSM-CNRS-Université Paris Diderot, Irfu/Service d'Astrophysique, CEA Saclay, Orme des Merisiers, \\ F-91191 Gif-sur-Yvette, France \\ ${ }^{4}$ DTU Space, National Space Institute, Technical University of Denmark, Elektrovej 327, DK-2800 Kgs. Lyngby, Denmark \\ ${ }^{5}$ Institute for Astronomy, Astrophysics, Space Applications and Remote Sensing, National Observatory of Athens, GR-15236 Athens, Greece \\ ${ }^{6}$ Dunlap Institute for Astronomy \& Astrophysics, 50 St. George Street, Toronto, ON M5S 3H4, Canada \\ ${ }^{7}$ European Southern Observatory, Karl-Schwarzschild-Straße 2, D-85748 Garching, Germany \\ ${ }^{8}$ Institute for Astronomy, University of Edinburgh, Royal Observatory, Blackford Hill, Edinburgh EH9 3HJ, UK \\ ${ }^{9}$ Department of Physics, Faculty of Science, University of Zagreb, Bijenicka cesta 32, 10000 Zagreb, Croatia \\ ${ }^{10}$ Leiden Observatory, Leiden University, P.O. Box 9513, 2300 RA Leiden, The Netherlands \\ ${ }^{11}$ Subdepartment of Astrophysics, University of Oxford, Keble Road, Oxford OX1 3RH, UK \\ Received 2019 June 7; revised 2019 August 28; accepted 2019 September 3; published 2019 November 22
}

\begin{abstract}
Optically compact star-forming galaxies (SFGs) have been proposed as immediate progenitors of quiescent galaxies, although their origin and nature are debated. Were they formed in slow secular processes or in rapid merger-driven starbursts? Answering this question would provide fundamental insight into how quenching occurs. We explore the location of the general population of galaxies with respect to fundamental star-forming and structural relations, identify compact SFGs based on their stellar core densities, and study three diagnostics of the burstiness of star formation: (1) star formation efficiency, (2) interstellar medium (ISM), and (3) radio emission. The overall distribution of galaxies in the fundamental relations points toward a smooth transition toward quiescence while galaxies grow their stellar cores, although some galaxies suddenly increase their specific star formation rate when they become compact. From their star formation efficiencies compact and extended SFGs appear similar. In relation to the ISM diagnostic, study of the CO excitation, the density of the neutral gas, and the strength of the ultraviolet radiation field shows that compact SFGs resemble galaxies located in the upper envelope of the main sequence of SFGs, although this is based on a small sample size. Regarding the radio emission diagnostic, we find that galaxies become increasingly compact as the starburst ages, implying that at least some compact SFGs are old starbursts. We suggest that compact SFGs could be starbursts winding down and eventually crossing the main sequence toward quiescence.
\end{abstract}

Key words: galaxies: bulges - galaxies: evolution - galaxies: formation - galaxies: fundamental parameters galaxies: high-redshift - galaxies: ISM

\section{Introduction}

In the past decade, various studies have revealed a tight correlation between the star formation rate (SFR) and the stellar mass of star-forming galaxies (SFGs). The so-called main sequence (MS) of star formation (e.g., Brinchmann et al. 2004; Daddi et al. 2007; Elbaz et al. 2007; Noeske et al. 2007; Whitaker et al. 2012) exhibits a small scatter observed at least up to $z \sim 4(\sim 0.3$ dex; e.g., Elbaz et al. 2007; Noeske et al. 2007; Whitaker et al. 2012; Speagle et al. 2014; Schreiber et al. 2015), implying that secular evolution is the dominant mode of stellar growth, where gas inflows, outflows, and consumption through star formation are in equilibrium (e.g., Daddi et al. 2010a; Genzel et al. 2010; Tacconi et al. 2010; Dekel et al. 2013; Feldmann \& Mayer 2015). Therefore, SFGs spend most of their time evolving as extended star-forming disks. Conversely, quiescent galaxies (QGs), having low specific star formation rate (sSFR), are located below the MS and are typically more compact than SFGs for a fixed stellar mass and redshift (e.g., van der Wel et al. 2014). The quenching of star formation and the departure from the MS must imply the buildup of a central stellar core (e.g., Kauffmann et al. 2003; Lang et al. 2014; van Dokkum et al. 2014; Barro et al. 2017a; Whitaker et al. 2017).
A population of galaxies have been proposed to be the missing link between the extended SFGs and the more compact QGs, the so-called compact star-forming galaxies (cSFGs; e.g., Barro et al. 2013, 2014; Nelson et al. 2014; Williams et al. 2014; van Dokkum et al. 2015). cSFGs are typically located within the scatter of the MS, although their origin and nature are still debated. Given the implications of the small scatter of the MS, several studies advocated that extended SFGs within the MS are capable of building up their stellar cores slowly in their secular evolution (e.g., Dekel et al. 2013; Zolotov et al. 2015; Tacchella et al. 2016). However, starburst galaxies (SBs) dominated by a violent episode of star formation typical of gasrich mergers that move well above the scatter of the MS are also capable of quickly building up compact stellar cores and have also been proposed as early progenitors of QGs (e.g., Cimatti et al. 2008; Ricciardelli et al. 2010; Fu et al. 2013; Ivison et al. 2013; Toft et al. 2014, 2017; Gómez-Guijarro et al. 2018).

Did the build-up of the stellar core, formation of cSFGs, and subsequent quenching of star formation happen as the product of the slow secular evolution or rapidly? Or in other words, is it the natural endpoint of secular galaxy evolution when a sufficiently large bulge has built up or does it require an external event such as a merger-induced starburst to compress 
the gas at the center of the collision and quickly convert it into stars?

Some works have recently pointed toward the starburst nature of cSFGs based on the properties of their interstellar medium (ISM) (e.g., Spilker et al. 2016; Barro et al. 2017b; Popping et al. 2017; Tadaki et al. 2017; Talia et al. 2018). However, these results are still based on a handful of cSFGs. Other recent works have also indicated the existence of a population of SBs within the scatter of the MS based on their high SFR surface densities, which are based on far-infrared (FIR) and radio observations (Elbaz et al. 2018; JiménezAndrade et al. 2019), and also a population of galaxies within the scatter of the MS undergoing compact star formation based on observations of CO lines (Puglisi et al. 2019).

In this work we explore the location of extended and compact SFGs and QGs with respect to the MS and structural relations, and investigate three diagnostics of the burstiness of star formation: (1) star formation efficiency (SFE), (2) ISM (CO excitation, density of the neutral gas, and strength of the ultraviolet field), and (3) radio emission (FIR/radio ratio and radio spectral slope). We aim to shed some light on how rapidly the build-up of compact stellar cores and subsequent quenching of star formation takes place.

The layout of the paper is as follows. We describe the sample selection and identification of extended SFGs, compact SFGs, and QGs in Section 2. In Section 3 we explore the distribution of SFGs and QGs with respect to fundamental star-forming and structural relations, which is followed by a discussion in Section 5. We investigate SFE, ISM, and radio emission diagnostics of the burstiness of star formation in Section 4. We summarize the main findings and conclusions in Section 6.

Throughout this work we adopted a concordance cosmology $\left[\Omega_{\Lambda}, \Omega_{M}, h\right]=[0.7,0.3,0.7]$ and Chabrier initial mass function (IMF) (Chabrier 2003).

\section{Selection of cSFGs}

\subsection{Optical Sample}

There are several selection criteria for CSFGs in the literature. We followed the identification criteria of Barro et al. (2017a) based on structural and star-forming relations. Barro et al. (2017a, see also references therein) showed that SFGs and QGs follow distinct trends in the plane of stellar density versus stellar mass, with QGs being offset to higher densities at fixed stellar mass and redshift. cSFGs are galaxies that follow the structural relation of QGs, while being star-forming. Therefore, cSFGs are more compact than regular SFGs at fixed stellar mass and redshift. Barro et al. (2017a) proposed a compactness selection threshold in the core density $\left(\Sigma_{1}, r<1 \mathrm{kpc}\right)$ as the most efficient way of selecting cSFGs, given the small scatter of the $\Sigma_{1}-M_{*}$ structural relation of QGs and the mild decline of normalization with redshift. This threshold is

$$
\Sigma_{1}-0.65\left(\log \left(M_{*} / M_{\odot}\right)-10.5\right)>\log B(z)-0.2,
$$

where $\log B(z)$ has a small redshift dependence ranging between 9.5 and $9.8 M_{*} \mathrm{kpc}^{-2}$ (see Barro et al. 2017a for details of its definition). For simplicity, we will refer to this threshold as $\Sigma_{1 \mathrm{QGs}}$ hereafter. In contrast, the structural relation of QGs based on the effective density $\left(\Sigma_{\mathrm{e}}, r<r_{\mathrm{e}}\right.$, where $r_{\mathrm{e}}$ is the effective radius) would be less efficient in identifying cSFGs because it shows larger scatter and variation of the normalization with redshift. By extension, other selection criteria based on stellar mass and effective radius would also be less efficient. Since by construction both cSFGs and QGs follow the same structural relation, the distinction between them is based on the distance to the main sequence of star formation ( $\triangle \mathrm{MS})$, defined as the ratio of the sSFR to the sSFR of the MS at the same stellar mass and redshift $\left(\triangle \mathrm{MS}=\mathrm{sSFR} / \mathrm{sSFR}_{\mathrm{MS}}\right.$ ). The threshold in Barro et al. (2017a) is $\Delta \mathrm{MS}=-0.7 \mathrm{dex}$, which corresponds to $\sim 2.5 \sigma$ below the MS. cSFGs are galaxies above this threshold in star formation.

For our analysis we worked with the cosmological fields COSMOS (Scoville et al. 2007) and GOODS-North (Dickinson et al. 2003). As a starting point, we employed the 3D-HST survey catalogs (Brammer et al. 2012; Skelton et al. 2014; Whitaker et al. 2014; Momcheva et al. 2016) in the CANDELS (Grogin et al. 2011; Koekemoer et al. 2011) portion of COSMOS and GOODS-North, from which we collected stellar masses, SFRs, and redshifts. The structural parameters were gathered from the associated catalogs in van der Wel et al. (2014). We trimmed the catalogs following Barro et al. (2017a): (1) $0.5<z<3.0$, to guarantee that the structural relations of Barro et al. (2017a) exist; (2) $\log \left(M_{*} / M_{\odot}\right)>9.0$ for SFGs, $\log \left(M_{*} / M_{\odot}\right)>10.0$ for QGs, and $H_{\mathrm{F} 160 \mathrm{~W}}<25.5$, to guarantee that the minimum requirements for the validity of the structural parameters are fulfilled (van der Wel et al. 2012, 2014), where sources flagged as catastrophic failures in the fits to surface brightness profiles were excluded. van der Wel et al. $(2012,2014)$ showed that the effective radius $\left(r_{\mathrm{e}}\right)$ and Sérsic index $(n)$ have uncertainties $<10 \%$ for galaxies with $H_{\mathrm{F} 160 \mathrm{w}}<24.5$, and discussed that a redshift-dependent mass threshold of $\log \left(M_{*} / M_{\odot}\right)>8.5-9.75$ for SFGs and $\log \left(M_{*} / M_{\odot}\right)>9.0-10.3$ for QGs at $0.5<z<3.0$ guarantees that the galaxies have $H_{\mathrm{F} 160 \mathrm{w}}<24.5$. Barro et al. (2017a) chose $\log \left(M_{*} / M_{\odot}\right)>9.0$ for SFGs and $\log \left(M_{*} / M_{\odot}\right)>10.0$ for QGs as a good compromise between dynamical range in stellar mass and accuracy in the structural parameters. To trace approximately the same rest-frame wavelength as a function of redshift we used the structural parameters derived in the $J_{\mathrm{F} 125 \mathrm{~W}}$ band at $z<1.5$ and the $H_{\mathrm{F} 160 \mathrm{~W}}$ band at $z \geqslant 1.5$. We refer to the sample resulting from this selection as our parent optical sample, which is composed of 13,703 galaxies (7222 in COSMOS and 6481 in GOODS-North) with 416 cSFGs (227 in COSMOS and 189 in GOODS-North).

\subsection{FIR Sample}

The "super-deblended" FIR to submillimeter photometric catalogs in COSMOS (Jin et al. 2018) and GOODS-North (Liu et al. 2018) provided fluxes from highly confused lowresolution data to optical counterparts by using a mix of priors based on bands with high spatial resolution (Spitzer/MIPS $24 \mu \mathrm{m}$, VLA $1.4 \mathrm{GHz}$ and $3 \mathrm{GHz}$ for COSMOS; Spitzer/MIPS $24 \mu \mathrm{m}$, VLA $1.4 \mathrm{GHz}$ for GOODS-North). We trimmed these catalogs to sources with a combined signal-to-noise ratio $\mathrm{S} / \mathrm{N}_{\mathrm{FIR}+\mathrm{mm}} \geqslant 5$ (where $\mathrm{S} / \mathrm{N}_{\mathrm{FIR}+\mathrm{mm}}$ is the sum in quadrature of the $\mathrm{S} / \mathrm{N}$ in all bands with $\lambda \geqslant 100 \mu \mathrm{m}$ in the catalogs (Liu et al. 2018; Jin et al. 2018)). We refer to the sample resulting from this selection as our FIR sample, which is composed of 968 galaxies (357 in COSMOS and 611 in GOODS-North) with 73 cSFGs (26 in COSMOS and 47 in GOODS-North).

\subsubsection{Rayleigh-Jeans (R-J) and Radio Subsets}

We separated a subset of galaxies in the FIR sample that have at least one detection at $\mathrm{S} / \mathrm{N} \geqslant 3$ above a rest-frame 
wavelength of $250 \mu \mathrm{m}$, the so-called R-J side of the FIR spectral energy distribution (SED), which are required to obtain gas mass estimates (Section 2.2.2). This comprises our R-J subset of the FIR sample, composed of 59 galaxies (24 in COSMOS and 35 in GOODS-North) with five cSFGs (four in COSMOS and one in GOODS-North).

Additionally, we cross-matched our FIR catalog with radio catalogs from the Giant Metrewave Radio Telescope at $325 \mathrm{MHz}$ and $610 \mathrm{MHz}$ in COSMOS (Tisanić et al. 2019) and at $610 \mathrm{MHz}$ in GOODS-North (Magnelli et al. 2015). Besides, we substituted the $3 \mathrm{GHz}$ measurements from the COSMOS "super-deblended" FIR catalog for those in the COSMOS-XS survey (D. van der Vlugt et al. 2019, in preparation; H. Algera et al. 2019, in preparation) for overlapping sources in both catalogs, given the increased depth of the latter survey. We looked for radio counterparts within the half-power beamwidth at each frequency. We separated a subset of galaxies that have at least two detections at $\mathrm{S} / \mathrm{N} \geqslant 5$ at any available radio frequency $(325 \mathrm{MHz}$, $610 \mathrm{MHz}, 1.4 \mathrm{GHz}$, and $3 \mathrm{GHz}$ in COSMOS; $610 \mathrm{MHz}$, $1.4 \mathrm{GHz}$, and $3 \mathrm{GHz}$ in GOODS-North), which are required for our radio diagnostic analysis (Section 4.3). This comprises our radio subset of the FIR sample, composed of 60 galaxies (23 in COSMOS and 37 in GOODS-North) with seven cSFGs (two in COSMOS and five in GOODS-North).

\subsubsection{FIR Properties}

We derived infrared luminosities $\left(L_{\mathrm{IR}}\right)$ and infrared-based star formation rates $\left(\mathrm{SFR}_{\mathrm{IR}}\right)$ for our FIR sample. In order to derive these quantities, we first fitted the mid-IR-to-millimeter SED using the models of Draine \& Li (2007). These models linearly combine two dust components, one coming from the diffuse ISM and one heated by a power-law distribution of starlight associated with photodissociation regions (PDRs). The methodology was presented in detail in previous studies (e.g., Magdis et al. 2012, 2017; Berta et al. 2016). We also included a component from active galactic nuclei (AGNs) to ensure that the derived FIR properties account for star formation only. The best fit to the models was derived through $\chi^{2}$ minimization and the uncertainties were calculated over 1000 realizations of the observed SED perturbing the photometry within the errors. $L_{\mathrm{IR}}$ was calculated by integrating the best fit to the SED in the range $8-1000 \mu \mathrm{m}$ and $\mathrm{SFR}_{\mathrm{IR}}$ from the $L_{\mathrm{IR}}$ to $\mathrm{SFR}_{\mathrm{IR}}$ conversion in Kennicutt (1998) for a Chabrier IMF. One of the parameters derived from the fit is the dust mass $\left(M_{\text {dust }}\right)$, which can be used to derive gas masses $\left(M_{\mathrm{gas}}\right)$. In order for the estimates of $M_{\mathrm{gas}}$ to be reliable, at least one detection on the R-J side of the SED is required. Therefore, we derive $M_{\text {gas }}$ only for our R-J subset of the FIR sample. We used the metallicity-dependent gas-todust mass ratio technique $\left(\delta_{\mathrm{GD}}\right)$, adopting the $M_{\mathrm{gas}} / M_{\mathrm{dust}}-Z$ relation of Magdis et al. (2012) $\left(\log \left(M_{\text {dust }} / M_{\text {gas }}\right)=(10.54 \pm\right.$ $1.0)-(0.99 \pm 0.12) \times(12+\log (\mathrm{O} / \mathrm{H})))$, where the gasphase metallicity is calibrated using the scale of Pettini \& Pagel (2004). We assumed a solar metallicity for all galaxies, which corresponds to $M_{\text {gas }} / M_{\text {dust }} \sim 90$. Another method to derive $M_{\text {gas }}$ is the single-band measurement of the dust continuum emission flux on the R-J side of the SED (e.g., Scoville et al. 2014, 2016; Groves et al. 2015; Schinnerer et al. 2016 ). Both the $\delta_{\mathrm{GD}}$ method and the method using a singleband measurement of the dust emission from Scoville et al. (2016) yielded consistent results on average, with a median and median absolute deviation ratio of $M_{\mathrm{gas}}^{\mathrm{GD}} / M_{\mathrm{gas}}^{\mathrm{R}-\mathrm{J}}=0.88 \pm 0.41$.
In the following we adopt estimates of $M_{\text {gas }}$ from the $\delta_{\mathrm{GD}}$ method, since it employs all data points in the SED, and particularly when there are several detections on the R-J side. Note that both methods account for the total gas budget of the galaxies, including the molecular $\left(M_{\mathrm{H}_{2}}\right)$ and the atomic $\left(M_{\mathrm{H}}\right)$ phases.

\subsection{Flagging of AGNs}

AGN activity is known to correlate with star formation (e.g., Kauffmann et al. 2003; Schawinski et al. 2010; Rodighiero et al. 2015) and to be present in a large fraction of cSFGs at $2<z<3$ (Barro et al. 2014). We kept track of the galaxies with evidence of AGN activity from several indicators for potential systematics in the AGN population with respect to the general population. We flagged all the galaxies for which the AGN fraction from our FIR SED modeling is $\geqslant 20 \%$. In addition, we checked for X-ray-bright AGNs $\left(\log L_{\mathrm{X}}>42.5\right.$, absorption-corrected soft and hard X-ray luminosity) in the COSMOS (Chandra COSMOS Legacy Survey; Civano et al. 2016; Marchesi et al. 2016) and GOODS-North (Xue et al. 2016) X-ray catalogs. Finally, we identified radio-excess AGNs as those having a significantly low FIR/radio ratio $(q<1.68)$ following Del Moro et al. (2013). These flagged AGNs account for unobscured to relatively obscured bright AGNs and radioloud AGNs, particularly for the FIR sample, for which all AGN indicators are available. We found that $22 \%$ of the massive $\left(\log \left(M_{*} / M_{\odot}\right) \geqslant 10.3\right)$ cSFGs have an AGN.

\section{Compactness and Star Formation}

In this section we explore the location of SFGs and QGs with respect to the MS of star formation and the structural relation of QGs.

For each galaxy in the parent optical sample we calculated $\Delta$ MS, adopting the MS definition of Whitaker et al. (2014), and the distance to the compactness selection threshold in the core density based on the structural relation of QGs $\left(\Delta \Sigma_{\mathrm{QGs}}=\right.$ $\Sigma_{1} / \Sigma_{1 \mathrm{QGs}}$ ), adopting the definition of the relation in Barro et al. (2017a), at its stellar mass and redshift. We will refer to extended SFGs as SFGs located at $\Delta \Sigma_{\mathrm{QGs}}<1.0$, as opposed to cSFGs located at $\Delta \Sigma_{\mathrm{QGs}}>1.0$. Similarly, extended QGs are QGs at $\Delta \Sigma_{\mathrm{QGs}}<1.0$ and compact $\mathrm{QGs}$ are $\mathrm{QGs}$ at $\Delta \Sigma_{\mathrm{QGs}}>1.0$.

Note that the SFRs in the 3D-HST catalogs are defined as $\mathrm{SFR}_{\mathrm{IR}+\mathrm{UV}}=1.09 \times 10^{-10}\left(L_{\mathrm{IR}}+2.2 L_{\mathrm{UV}}\right)$, where $L_{\mathrm{IR}}$ is obtained through a conversion from the observed Spitzer/ MIPS $24 \mu \mathrm{m}$ flux density to $L_{\mathrm{IR}}(8-1000 \mu \mathrm{m})$ based on a single template. $L_{\mathrm{UV}}$ is the total integrated rest-frame luminosity in the range 1216-3000 $\AA$. For the FIR sample ( $7 \%$ of the parent optical sample) we substituted the $\mathrm{SFR}_{\mathrm{IR}}$ contribution for the one we obtained in Section 2.2.2, since it uses all the information available in the FIR SED, as opposed to a single template, which could dilute galaxies that intrinsically deviate from it. We checked that making this $\mathrm{SFR}_{\mathrm{IR}}$ substitution does not introduce a systematic bias with respect to the MS definition of Whitaker et al. (2014), which could alter our $\Delta \mathrm{MS}$ values.

\subsection{General Trends}

In Figure 1 we present the $\Delta \mathrm{MS}-\Delta \Sigma_{\mathrm{QGs}}$ plane for the parent optical sample. The overall distribution reproduces the L-shape reported in Barro et al. (2017a), with the population of cSFGs 

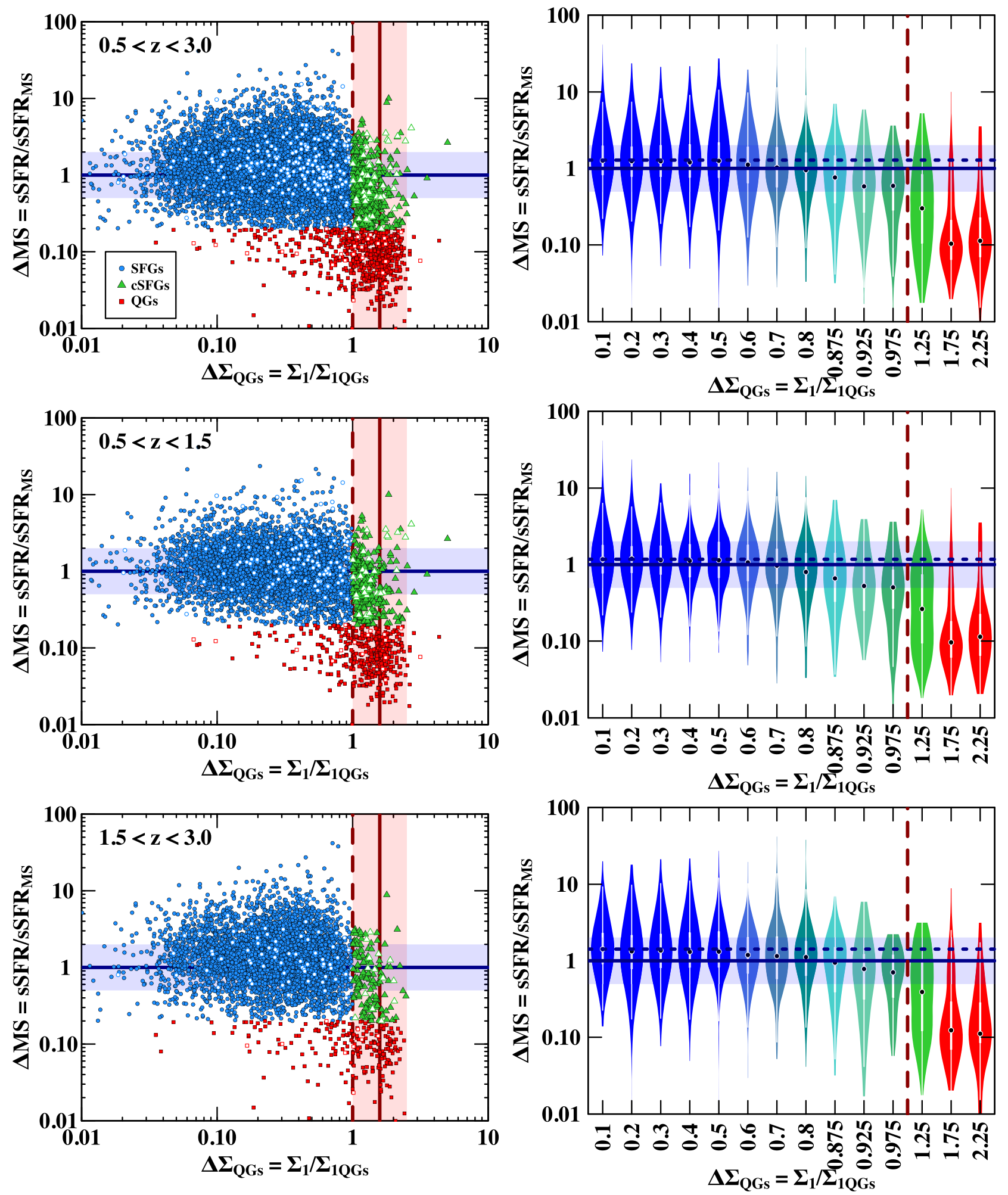

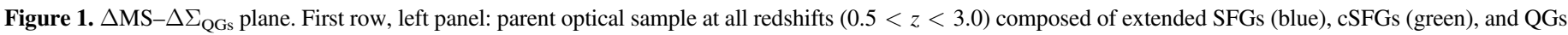

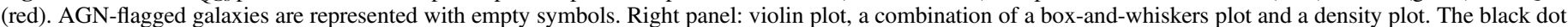

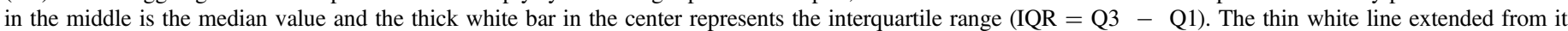

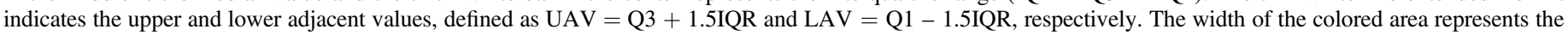

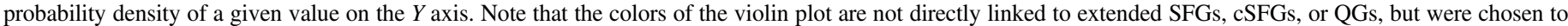

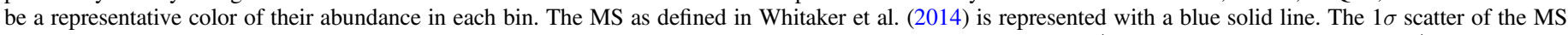

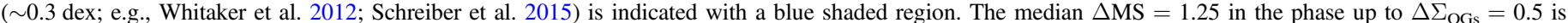

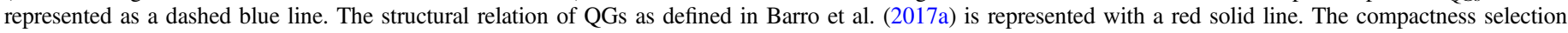

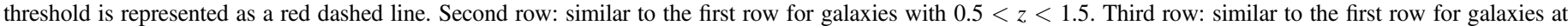
$1.5<z<3.0$. The typical uncertainties are 0.15 dex on the $X$ axis and 0.10 dex on the $Y$ axis. 
forming the knee between extended SFGs and compact QGs. This was used as an argument in favor of cSFGs as progenitors of QGs at later times, implying that SFGs become compact before they quench. Note that the majority of QGs are compact.

We also explore the behavior of $\Delta \mathrm{MS}$ ( $Y$ axis) per bin of $\Delta \Sigma_{\mathrm{QGs}}(X$ axis) in Figure 1. In order to do so, we draw a violin plot - a combination of a box-and-whiskers plot and a density plot- to visualize the distribution of the data and its probability density. The violin plot has the advantage of showing not only a discrete median value per bin on the $X$ axis, but also different measurements of the scatter and the actual shape of the distribution of the data on the $Y$ axis.

Overall, galaxies start to transition smoothly toward quiescence, since $\Delta \mathrm{MS}$ decreases continuously for increasing $\Delta \Sigma_{\mathrm{QGs}}$. Some extended SFGs quench, forming extended QGs as they build up their stellar cores. On the other hand, the sharp transition region at $\Delta \Sigma_{\mathrm{QGs}} \sim 1.0$ indicates that other galaxies become compact before they quench, as reported in Barro et al. (2017a). Some extended SFGs become cSFGs and then compact QGs as they build up their stellar cores. The latter would be a more common track since the majority of QGs are compact. Note that it has to be considered that SFGs do not evolve into QGs at the same epoch (i.e., redshift), but at later times.

The behavior around the transition threshold at $\Delta \Sigma_{\mathrm{QGs}} \sim$ 1.0 presents some remarkable features. In the bin centered at $\Delta \Sigma_{\mathrm{QGs}}=1.25$, while the median $\Delta \mathrm{MS}$ decreases abruptly, reflecting the sharp transition region mentioned above, the scatter in $\triangle \mathrm{MS}$ increases in both directions of the $Y$ axis. In particular, it is interesting that the upper extreme values increase with respect to the previous bin centered at $\Delta \Sigma_{\mathrm{QGs}}=$ 0.975 . Even in the bin centered at $\Delta \Sigma_{\mathrm{QGs}}=1.75$ the upper extreme values still increase, although they are less frequent than in the previous bin. This indicates that at least some of the galaxies make the transition by increasing their sSFR and going above the scatter of the MS.

Furthermore, it is also interesting that the median $\Delta \mathrm{MS}$ stays approximately constant up to $\Delta \Sigma_{\mathrm{QGs}}=0.5$ and systematically above the MS ( $\triangle \mathrm{MS}=1.25 ; \sim 0.1 \mathrm{dex}$ ). This indicates that, while the MS is dominated by galaxies in the extended phase, there is a contribution from more compact galaxies in transition toward quiescence that affects the overall trend that defines the MS, lowering its normalization. Another interesting fact is that extended SFGs above the scatter of the MS are far more numerous than cSFGs above the scatter of the MS.

\subsection{Redshift Dependence}

In addition to the $\Delta \mathrm{MS}-\Delta \Sigma_{\mathrm{QGs}}$ plane for the whole redshift range studied, we also present the results in the redshift bins $0.5<z<1.5$ and $1.5<z<3.0$ in Figure 1. The general trends are similar at low and high redshift, although there are some important differences. At $1.5<z<3.0$ the median $\Delta \mathrm{MS}$ up to $\Delta \Sigma_{\mathrm{QGs}}=0.5$ is higher $(\Delta \mathrm{MS}=1.35)$ than at $0.5<z<1.5(\Delta \mathrm{MS}=1.16)$. This indicates that the MS is more affected in the high-redshift bin than in the low-redshift bin by galaxies that are already in transition toward quiescence and that lower its normalization.

\subsection{Trends for Massive Galaxies}

The $\Delta \mathrm{MS}-\Delta \Sigma_{\mathrm{QGs}}$ planes discussed above follow the selection criteria explained in Section 2.1. In particular, the stellar mass limits are $\log \left(M_{*} / M_{\odot}\right)>9.0$ for SFGs, $\log \left(M_{*} / M_{\odot}\right)>10.0$ for QGs. At $\log \left(M_{*} / M_{\odot}\right) \geqslant 10.3$ the sample is complete for both SFGs and QGs at $z<3.0$ (van der Wel et al. 2014; see also Skelton et al. 2014; Tal et al. 2014; Barro et al. 2017a). Therefore, we explored the $\Delta \mathrm{MS}-\Delta \Sigma_{\mathrm{QGs}}$ plane for the most massive galaxies with $\log \left(M_{*} / M_{\odot}\right) \geqslant 10.3$ in Figure 2.

Overall, the trends are similar to those discussed in the previous section. Note that the sample statistics are smaller in this case, which has to be taken into consideration when interpreting the plots. One important difference is that the median $\triangle \mathrm{MS}$ is no longer approximately constant up to $\Delta \Sigma_{\mathrm{QGs}}=0.5$, but rather starts to decay after the first bin centered at $\Delta \Sigma_{\mathrm{QGs}}=0.1$. This is expected as a consequence of massive galaxies being more dominated by galaxies that are already in transition toward quiescence than low-mass galaxies. In this case we do not appreciate differences in $\triangle \mathrm{MS}$ at $\Delta \Sigma_{\mathrm{QGs}}=0.1$ at low redshift $(\Delta \mathrm{MS}=1.27)$ and high redshift $(\Delta \mathrm{MS}=1.25)$, which indicates that the trend in Section 3.1 was dominated by low-mass galaxies. Another important difference is that the number of galaxies above the scatter of the MS as opposed to within the scatter is smaller for massive galaxies. Besides, outliers are less strong (i.e., smaller $\Delta \mathrm{MS}$ ), as expected given that for the same increase in SFR the effect on sSFR is smaller as galaxies become more massive.

\section{Are cSFGs Normal SFGs or Starbursts?}

cSFGs have been proposed as a transition population between being star-forming and quiescence (e.g., Barro et al. 2013, 2014; Nelson et al. 2014; Williams et al. 2014; van Dokkum et al. 2015). Revealing their nature implies revealing whether the transition to quiescence occurred secularly or rapidly. Phases of abrupt changes in increasing sSFR are typical of SBs. The time a galaxy is detectable in such a phase is short, since they are short-lived (e.g., Di Matteo et al. 2008; Tacconi et al. 2008). This means that the number of detectable SBs is small compared to the general population, but the phase can still be very relevant in terms of stellar mass assembly. We examined three diagnostics of the burstiness of star formation: (1) SFE, (2) ISM, and (3) radio emission. The aim is to explore whether cSFGs can be considered as normal SFGs, pointing to a more secular evolution, or as SBs, pointing to a more rapid evolution. We refer to normal SFGs as those that obey the general trends of SFGs in each diagnostic. Conversely, SBs are outliers to these general trends in each diagnostic (see the sections on diagnostics for a more detailed explanation). These definitions of normal SFGs and SBs are independent of their position with respect to the MS. The latter would be a consequence of the physical mechanisms in place (related to our diagnostics) and the effect of these mechanisms on the integrated properties of the galaxies at the time of observation. Note also that the galaxies selected for each of the three diagnostics are not the same sources because the selections do not overlap.

\subsection{Diagnostic 1: SFE}

The star formation law or Kennicutt-Schmidt (KS) relation (Schmidt 1959; Kennicutt 1998) relates the gas mass and the SFR of SFGs (originally defined using surface densities). Several studies indicated that normal SFGs and SBs follow different trends. SBs have higher SFR per unit of gas mass, and 

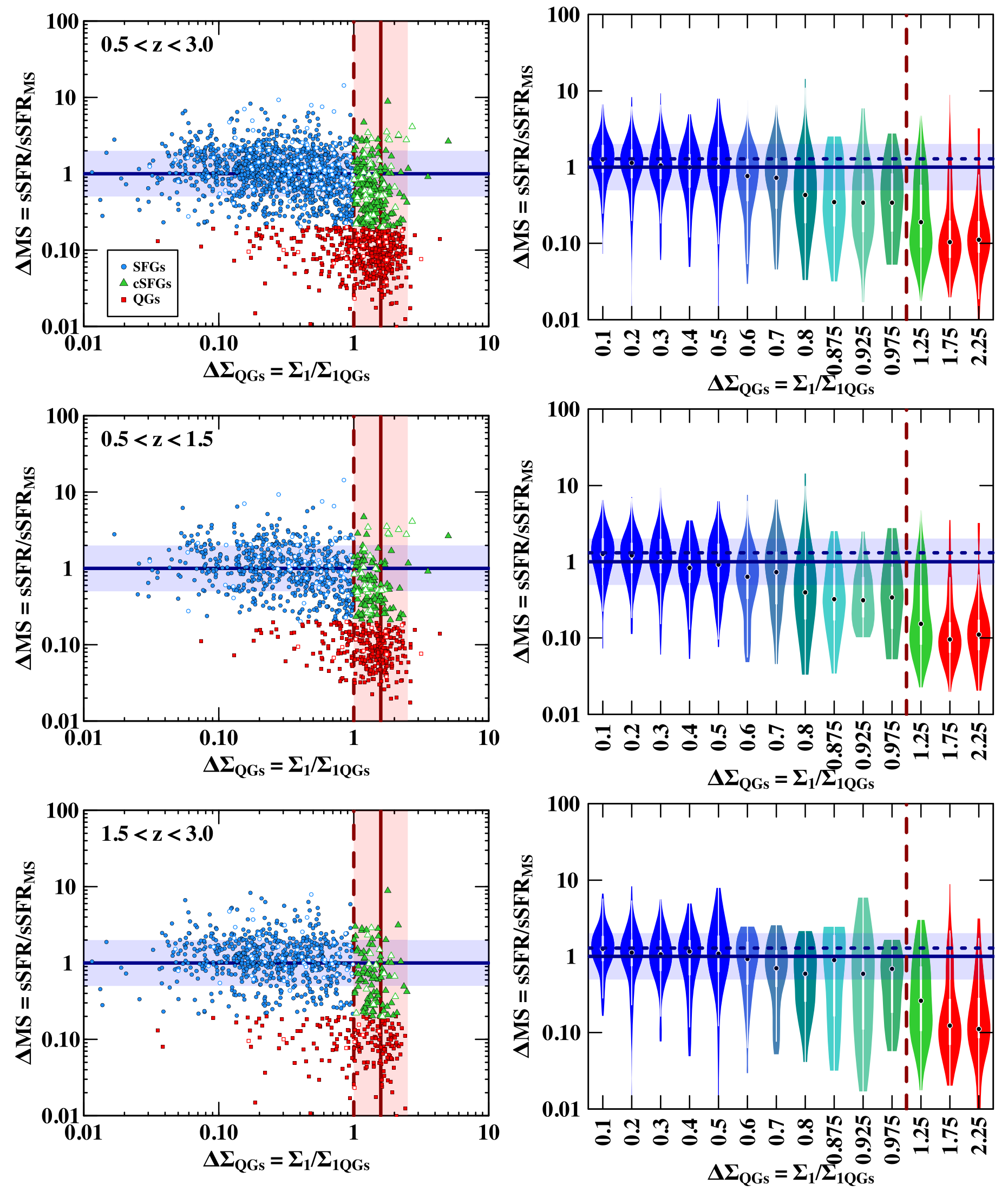

Figure 2. $\Delta \mathrm{MS}-\Delta \Sigma_{\mathrm{QGs}}$ plane as presented in Figure 1 for galaxies with $\log \left(M_{*} / M_{\odot}\right) \geqslant 10.3$.

thus higher star formation efficiencies $\left(\mathrm{SFE}=\mathrm{SFR} / M_{\mathrm{gas}}\right)$ than normal SFGs (e.g., Daddi et al. 2010b; Genzel et al. 2010). This distinction in SFE serves to distinguish normal SFGs from
SBs, regardless of their location with respect to the MS. In this section we apply this SFE-based definition of normal SFGs and SBs. 

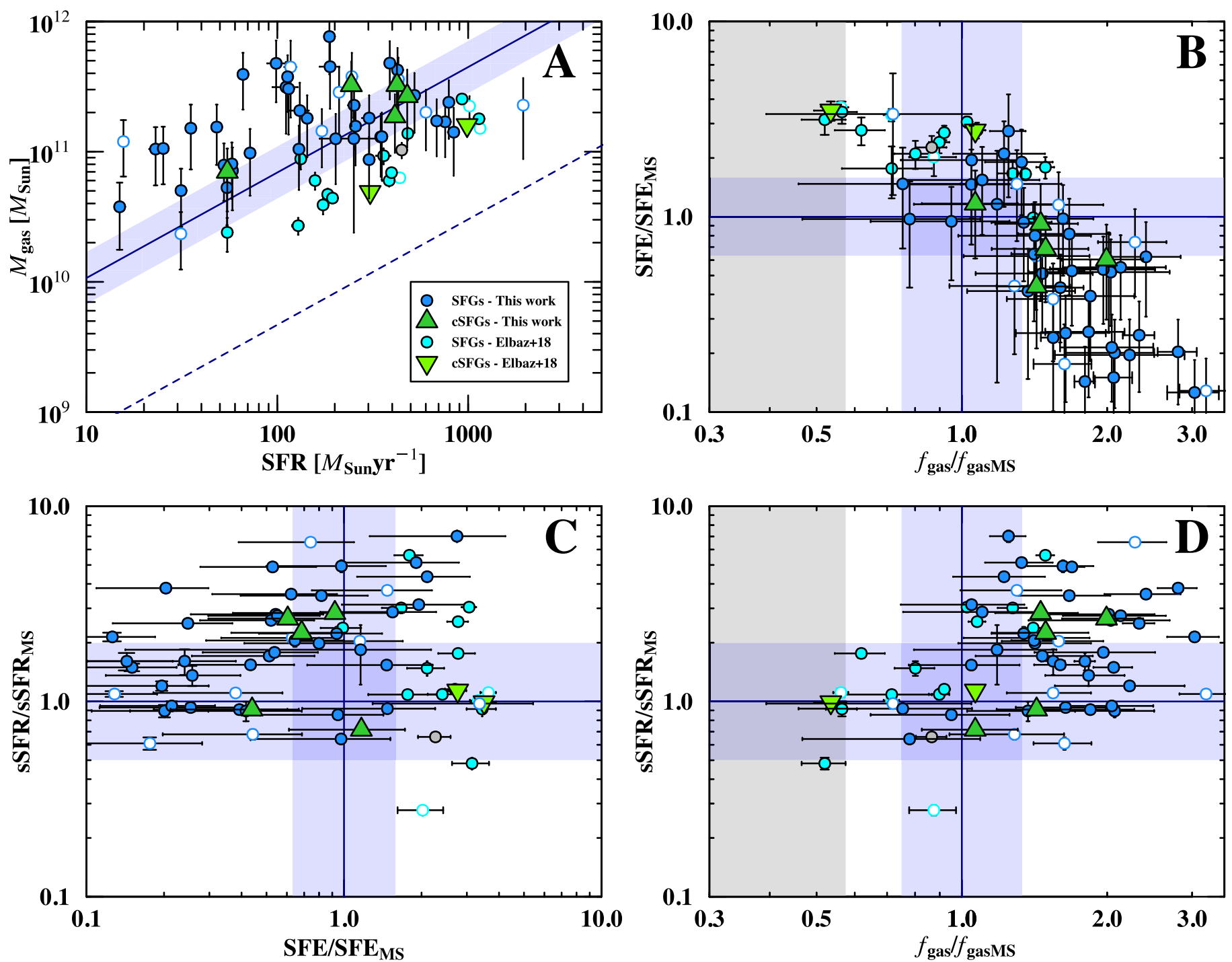

Figure 3. A: $M_{\text {gas }}-$ SFR plane. The trend for normal SFGs (solid line), with its 0.2 dex scatter, and that for SBs (dashed line) from Sargent et al. (2014) are shown for reference. B: SFE- $f_{\text {gas }}$ plane. C: $\Delta \mathrm{MS}-\mathrm{SFE}$ plane. D: $\Delta \mathrm{MS}-f_{\text {gas }}$ plane. SFE and $f_{\text {gas }}$ are normalized to the trend for normal SFGs in Sargent et al. (2014). The $1 \sigma$ scatter of the MS ( $\sim 0.3$ dex; e.g., Whitaker et al. 2012; Schreiber et al. 2015) and the trends for SFE ( $\sim 0.2$ dex; Sargent et al. 2014) and $f_{\text {gas }}(\sim 0.125$ dex; Sargent et al. 2014) in normal SFGs are represented as blue shaded regions. Our sample and that of Elbaz et al. (2018) are shown, classified as extended SFGs (blue circles) or cSFGs (green triangles), except for one of galaxy of Elbaz et al. (2018) (gray circle) that is unclassifiable due to bad structural parameters. AGN-flagged galaxies are represented with empty symbols. In panels $\mathrm{B}$ and $\mathrm{D}$ the most favorable $f_{\text {gas }}$ limit in our selection is shown as a gray shaded region as a reference for the detection threshold (see Section 5).

In Figure 3 we present the locus of our R-J subset of the FIR sample in the $M_{\mathrm{gas}}-\mathrm{SFR}$ plane in relation to the trends for normal SFGs and SBs in Sargent et al. (2014), where the trend for normal SFGs comes from massive MS galaxies in Sargent et al. (2014). Note that we only included the most massive subset of galaxies with $\log \left(M_{*} / M_{\odot}\right) \geqslant 10.3$, to guarantee that the assumption of solar metallicity in deriving $M_{\text {gas }}$ is valid. We also included the sample of Elbaz et al. (2018), calculating their $\Delta \Sigma_{\mathrm{QGs}}$ and assessing whether they are extended SFGs or cSFGs, according to the criterion of Barro et al. (2017a). This can be successfully done for 18/19 galaxies in Elbaz et al. (2018) because one of the galaxies has bad structural parameters in the catalogs of van der Wel et al. (2014) (shown with a gray symbol in Figure 3). In Figure 3 we also explore the relation between SFE, gas fraction $\left(f_{\text {gas }}=M_{\text {gas }} /\left(M_{*}+M_{\text {gas }}\right)\right.$ ), and $\triangle \mathrm{MS}$, where SFE and $f_{\text {gas }}$ are normalized to the trends for normal SFGs as defined by the trends for MS galaxies in Sargent et al. (2014).
cSFGs in our sample are consistent with the trend in SFE established for normal SFGs. Besides, the extended SFGs in our sample follow the trend in SFE in normal SFGs as well. Our sample is located within and above the scatter of the MS. The SFE $-f_{\text {gas }}$ plane exhibits a tendency for galaxies with lower SFE to be those with higher $f_{\text {gas }}$, as expected for galaxies that decrease their SFE as a consequence of increasing their gas content. On the other hand, the sample of Elbaz et al. (2018) occupies complementary regions in these diagrams to our sample, exhibiting enhanced SFE closer to those of the trend for SBs due to low gas fractions. Although beyond the scope of our work, the $\Delta$ MS-SFE and $\Delta \mathrm{MS}-f_{\text {gas }}$ planes reflect the general trends of SFE and $f_{\text {gas }}$ to increase with $\triangle \mathrm{MS}$, as reported in the literature (e.g., Scoville et al. 2017; Tacconi et al. 2018).

These diagrams indicate that our cSFGs are consistent with the trends of normal SFGs with no evidence of SB-like SFE. The combination of our sample with that of Elbaz et al. (2018) 

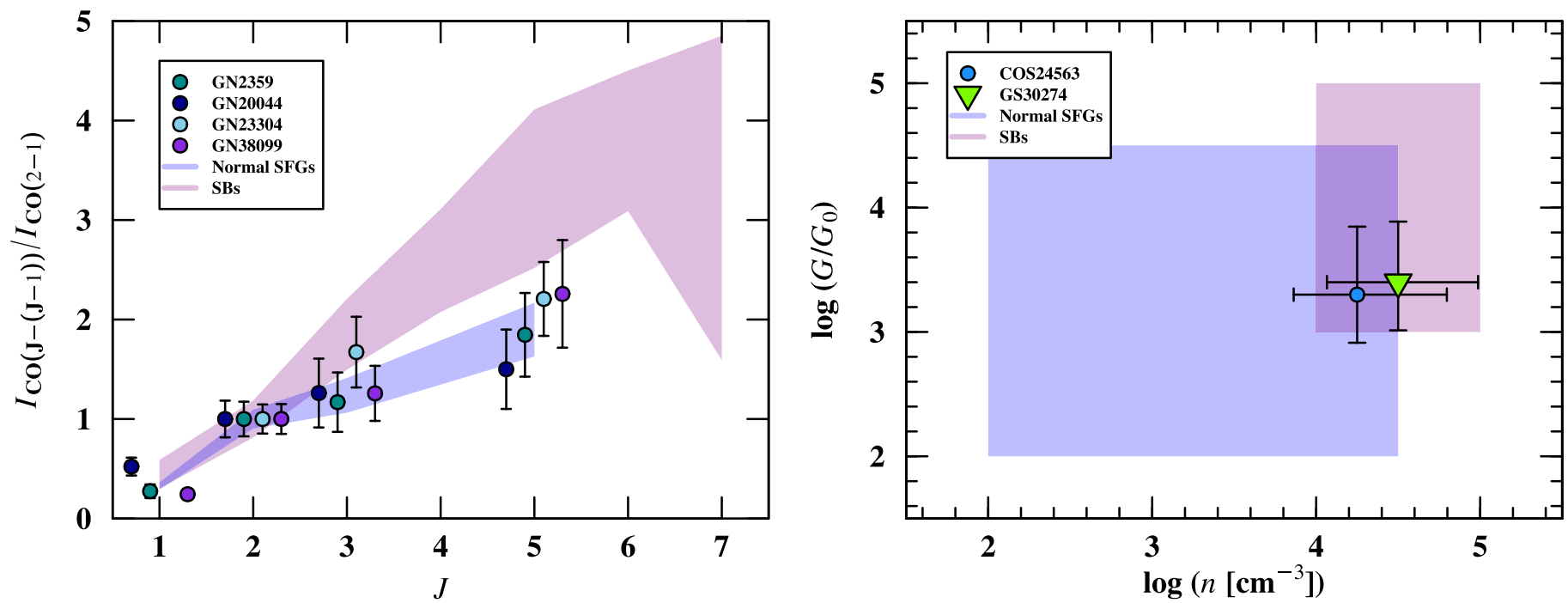

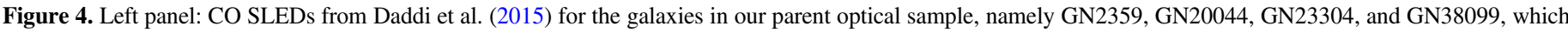

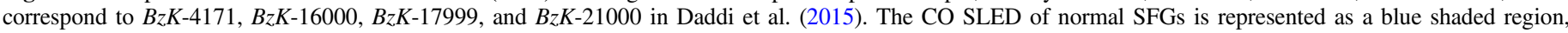

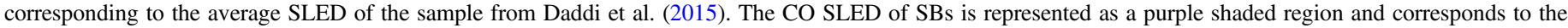

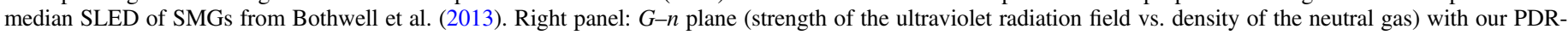

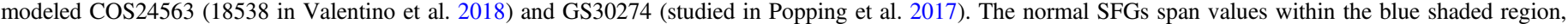

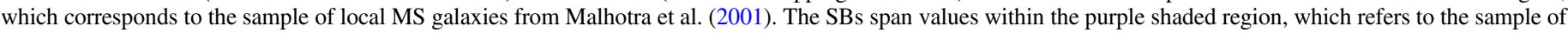
local ULIRGS from Davies et al. (2003).

indicates that there is no difference between cSFGs and extended SFGs in terms of their SFE, since both occupy the same regions in the SFE and $f_{\text {gas }}$ parameter space.

Overall, these results based on the relations among $M_{\mathrm{gas}}$, stellar mass, and SFR point toward both secular (lower SFE) and rapid (higher SFE) evolution processes being able to generate cSFGs.

\subsection{Diagnostic 2: ISM}

\subsubsection{CO Excitation}

The properties of the ISM are a critical piece of information in studying how star formation occurs. The excitation of $\mathrm{CO}$ emission is a good tracer of the ISM properties. It is measured through the line luminosity ratio of $\mathrm{CO}$ lines with different rotational numbers $(J)$. The $\mathrm{CO}(5-4) / \mathrm{CO}(2-1)$ ratio shows the biggest discrepancy between normal SFGs and SBs than any other pair of $\mathrm{CO}$ transitions calibrated in the literature (e.g., Bothwell et al. 2013; Daddi et al. 2015). Daddi et al. (2015) established a benchmark for the excitation conditions of normal SFGs by studying a sample of $B z K$-selected SFGs at $z \sim 1.5$ located within the scatter of the MS. They found that, while less excited than typical SBs such as local ultraluminous infrared galaxies (ULIRGs) or high-redshift submillimeter galaxies (SMGs), the average excitation was higher than in the Milky Way. The authors argued that the excitation correlates with the star formation surface density. This, along with the fact that the excitation varied within the sample, motivated us to study whether some of the galaxies of Daddi et al. (2015) are cSFGs. In this section we refer to normal SFGs as those consistent with the $\mathrm{CO}$ spectral line energy distribution (SLED) of the $B z K$-selected MS galaxies in Daddi et al. (2015), while we refer to SBs as those consistent with the median CO SLED of SMGs from Bothwell et al. (2013).

We cross-matched our parent optical sample with the galaxies in Daddi et al. (2015). We found that three of our galaxies, namely GN2359, GN20044, and GN23304, correspond to $B z K-4171$,
Table 1

$\Delta \mathrm{MS}$ and $\Delta \Sigma_{\mathrm{QGs}}$ for Galaxies in Section 4.2

\begin{tabular}{lcc}
\hline \hline Name & $\Delta$ MS & $\Delta \Sigma_{\text {QGs }}$ \\
\hline COS24563 & $3.15 \pm 0.12$ & $0.325 \pm 0.011$ \\
GN2359 & $2.81 \pm 0.11$ & $0.227 \pm 0.012$ \\
GN20044 & $1.13 \pm 0.08$ & $0.394 \pm 0.007$ \\
GN23304 & $2.37 \pm 0.05$ & $0.227 \pm 0.012$ \\
GN38099 & $5.13 \pm 0.10$ & $0.095 \pm 0.003$ \\
GS30274 & $2.02 \pm 0.10$ & $1.132 \pm 0.011$ \\
\hline
\end{tabular}

$B z K-16000$, and $B z K-17999$ in Daddi et al. (2015), respectively. The missing galaxy $B z K-21000$ in Daddi et al. (2015) corresponds to GN38099. Its structural parameters are poorly constrained and thus it was excluded from our sample. For the analysis in this section we added it back, bearing in mind this caveat. In Figure 4 we show the SLEDs in Daddi et al. (2015) for these four galaxies. In Table 1 we present their $\Delta \mathrm{MS}$ and $\Delta \Sigma_{\mathrm{QGs}}$ values.

All four galaxies are extended SFGs and not cSFGs. However, we see that the three galaxies with the highest $\Delta \mathrm{MS}$ are the ones with the highest CO excitation (GN2359, GN23304, and GN38099), while the other galaxy located right on the MS has the lowest CO excitation (GN20044).

The galaxies with the highest $\mathrm{CO}$ excitation are also those with the highest star formation surface density according to Daddi et al. (2015), suggesting that the scatter at higher $\Delta \mathrm{MS}$ is linked to galaxies progressively forming compact cores.

\subsubsection{Photodissociation Regions}

Another way of studying the ISM properties is through characterizing the emission from PDRs. PDRs are regions of neutral gas dominated by far-ultraviolet (FUV) photons. PDR modeling has been used to characterize the strength of the ultraviolet radiation field $(G)$ and the density of the neutral gas (n) (e.g., Alaghband-Zadeh et al. 2013; Popping et al. 2017). In particular, Popping et al. (2017) employed it to characterize 
the ISM properties of a cSFG at $z=2.225$ located within the scatter of the MS (namely GS30274). They found that the galaxy has SB-like ISM properties, low gas fraction, and high efficiency compared to normal SFGs. The authors interpreted this to show that a previous merger event triggered a central starburst that is quickly building up a dense core of stars responsible for the compact distribution of stellar light. We studied whether galaxies in our sample are similar. In this section we refer to normal SFGs as those consistent with the location of the sample of MS galaxies from Malhotra et al. (2001) in the $G-n$ plane, while we refer to SBs as those consistent with the location of the sample of local ULIRGS from Davies et al. (2003) in the $G-n$ plane, identical to the definition used in Alaghband-Zadeh et al. (2013) and Popping et al. (2017).

Valentino et al. (2018) presented a survey of atomic carbon [C I] of FIR-selected galaxies on the MS at $z \sim 1.2$. We crossmatched our parent optical sample with the galaxies in Valentino et al. (2018). We found that one of our extended SFGs was observed in that survey, namely COS24563 (which corresponds to 18538 in Valentino et al. 2018). We performed PDR modeling for this galaxy and also for GS30274 in Popping et al. (2017) for consistency in the methodology and to avoid systematics in the comparison. Besides, we calculated $\Delta \Sigma_{\mathrm{QGs}}$ for GS30274 and checked that it is a cSFG according to the criterion of Barro et al. (2017a). Note that the structural parameters of GS30274 are poorly constrained, as also mentioned in Popping et al. (2017), and do not meet the quality criteria applied to our sample. In Figure 4 we locate the two modeled galaxies in the $G-n$ plane. In Table 1 we present their $\Delta \mathrm{MS}$ and $\Delta \Sigma_{\mathrm{QGs}}$ values.

We estimated the density $n$ (in $\mathrm{cm}^{-3}$ ) and the strength of the FUV $(6 \mathrm{eV}<h \nu<13.6 \mathrm{eV})$ radiation field $G$ (in Habing field units, $G_{0}=1.6 \times 10^{-3} \mathrm{erg} \mathrm{cm}^{-2} \mathrm{~s}^{-1}$ ) by comparing the available line luminosities with the 1D modeling of the PDRs by Kaufman et al. (1999, 2006). This modeling provides a simplified picture of the complex cold ISM phases and their interplay in high-redshift galaxies, but it is enough to capture the average properties of these unresolved systems, without introducing a large number of parameters that cannot be observationally constrained at the current stage. We downloaded the relevant line intensity maps from the online PDR TOOLBOX (Pound \& Wolfire 2008), originally spanning a density interval of $1<\log n\left(\mathrm{~cm}^{-3}\right)<7$ and FUV intensity range of $-0.5<\log \left(G / G_{0}\right)<6.5$, and we resampled them to a grid with steps of 0.05 dex. We then compared the models and the observations, finding the combination of $(n, G)$ that minimizes $\chi^{2}$. We estimated the uncertainties on the best fit $(n, G)$, both applying the criterion described in Avni (1976) and bootstrapping 1000 times the line luminosities, and computed the $68 \%, 90 \%$, and $95 \%$ confidence intervals as interpercentile ranges. In this work we modeled the ${ }^{3} P_{1} \rightarrow{ }^{3} P_{0}$ transition of neutral atomic carbon $\left([\mathrm{C} \mathrm{I}]\left({ }^{3} P_{1}-{ }^{3} P_{0}\right), \nu_{\text {rest }}=492.161 \mathrm{GHz}\right)$, a mid- $J$ CO line $\left(\mathrm{CO}(4-3)\right.$ or $\mathrm{CO}(5-4)$ at $\nu_{\text {rest }}=461.0408$ and $576.2679 \mathrm{GHz}$, respectively), and the total infrared luminosity $\left(L_{\mathrm{IR}}\right)$, removing the possible AGN contribution due to the dusty torus around the central supermassive black hole (see Section 2.2.2). The $[\mathrm{C} \mathrm{I}]\left({ }^{3} P_{1}-{ }^{3} P_{0}\right) /$ mid- $J$ ratio is primarily sensitive to the density. The use of $\mathrm{CO}(4-3)$ or $\mathrm{CO}(5-4)$ as the CO mid- $J$ transition does not affect the results for the density (F. Valentino et al. 2019, in preparation). [C I] $\left({ }^{3} P_{1}-{ }^{3} P_{0}\right) / L_{\mathrm{IR}}$ depends on $G$ by construction ( $G \propto L_{\mathrm{IR}}$, Kaufman et al. 1999). We thus have roughly perpendicular tracks to determine both $(n, G)$ parameters (e.g., Alaghband-Zadeh et al. 2013; Popping et al. 2017).

COS24563 and GS30274 have similar properties, as seen in Figure 4. The ISM properties of both are located at the intersection between those of normal SFGs and those of SBs. COS24563 is placed at a $\Delta \mathrm{MS}$ slightly above the scatter of the MS, suggesting that the scatter at higher $\Delta \mathrm{MS}$ is linked to galaxies progressively forming compact cores, similar to the interpretation drawn from the $\mathrm{CO}$ excitation.

Overall, the ISM properties from both the $\mathrm{CO}$ excitation and PDR modeling suggest that extended SFGs located slightly above the MS (upper-MS galaxies) are capable of hosting an ISM that appears mildly excited and dense, similar to the lower envelope of SB-like ISM properties. This suggests that the build-up of a compact core leading to cSFGs could happen secularly, or at least that, if coming from the product of a rapid starburst-like event, the latent ISM has similar properties to that of upper-MS normal SFGs. However, the small sample sizes and the lack of ISM characterization of CSFGs in this work and over the literature makes it is still difficult to conclude whether the shown ISM properties are the product of a slow secular evolution or the final stages of SBs pointing toward a more rapid evolution.

\subsection{Diagnostic 3: Radio Emission}

The FIR/radio correlation (FRC; e.g., de Jong et al. 1985; Helou et al. 1985; Condon 1992) arises because massive stars $\left(M_{*}>8 M_{\odot}\right)$ are responsible for producing ultraviolet photons that are absorbed and re-emitted by dust at FIR wavelengths, and also responsible for accelerating cosmic-ray electrons after exploding as supernovae, which produces synchrotron emission at radio wavelengths. Bressan et al. (2002) modeled the FIR and radio emission of SBs, studying the interplay between the two with the age of the starburst episode and their effects on the FIR/radio ratio $\left(q \propto L_{\mathrm{IR}} / L_{\text {radio }}\right)$ and on the slope of the power-law radio spectrum $\left(S \propto \nu^{-\alpha}\right)$; they introduced the $q_{1.4 \mathrm{GHz}}-\alpha$ diagram as a diagnostic of the evolution of SBs. During the very early phase after star formation ignites, SBs are dominated by FIR emission since only thermal free-free emission from $\mathrm{H}$ II regions contributes to the radio emission. At this stage the radio slope is shallow $(\alpha \sim 0.2)$ and the radio output is low compared to FIR $\left(q_{1.4 \mathrm{GHz}} \sim 3\right)$. Then, corecollapse supernovae explosions occur, feeding relativistic electrons to the galactic magnetic fields, and non-thermal synchrotron emission steepens the radio spectrum, increasing the radio output at the same time. At this stage the radio slope progressively becomes similar to the value typical of normal SFGs $(\alpha \sim 0.8)$ and the $\mathrm{FIR} /$ radio ratio progressively decreases to a minimum value $\left(q_{1.4 \mathrm{GHz}} \sim 1.7\right)$. At older ages, the FIR/radio ratio increases again at almost constant radio slope ( $\alpha$ and $q_{1.4 \mathrm{GHz}}$ reach asymptotic values). These models were first observationally tested by Thomson et al. (2014) for $870 \mu \mathrm{m}$ selected SMGs. The authors found that the data populated the predicted region of the parameter space and the stellar masses tend to increase along the evolutionary tracks of SBs in the $q_{1.4 \mathrm{GHz}}-\alpha$ diagram. We explored the location of our galaxies in this diagram as another diagnostic of their nature. In this section we refer to normal SFGs as having typical average values for SFGs of $\alpha=0.80 \pm 0.25$ (e.g., Condon 1992; 


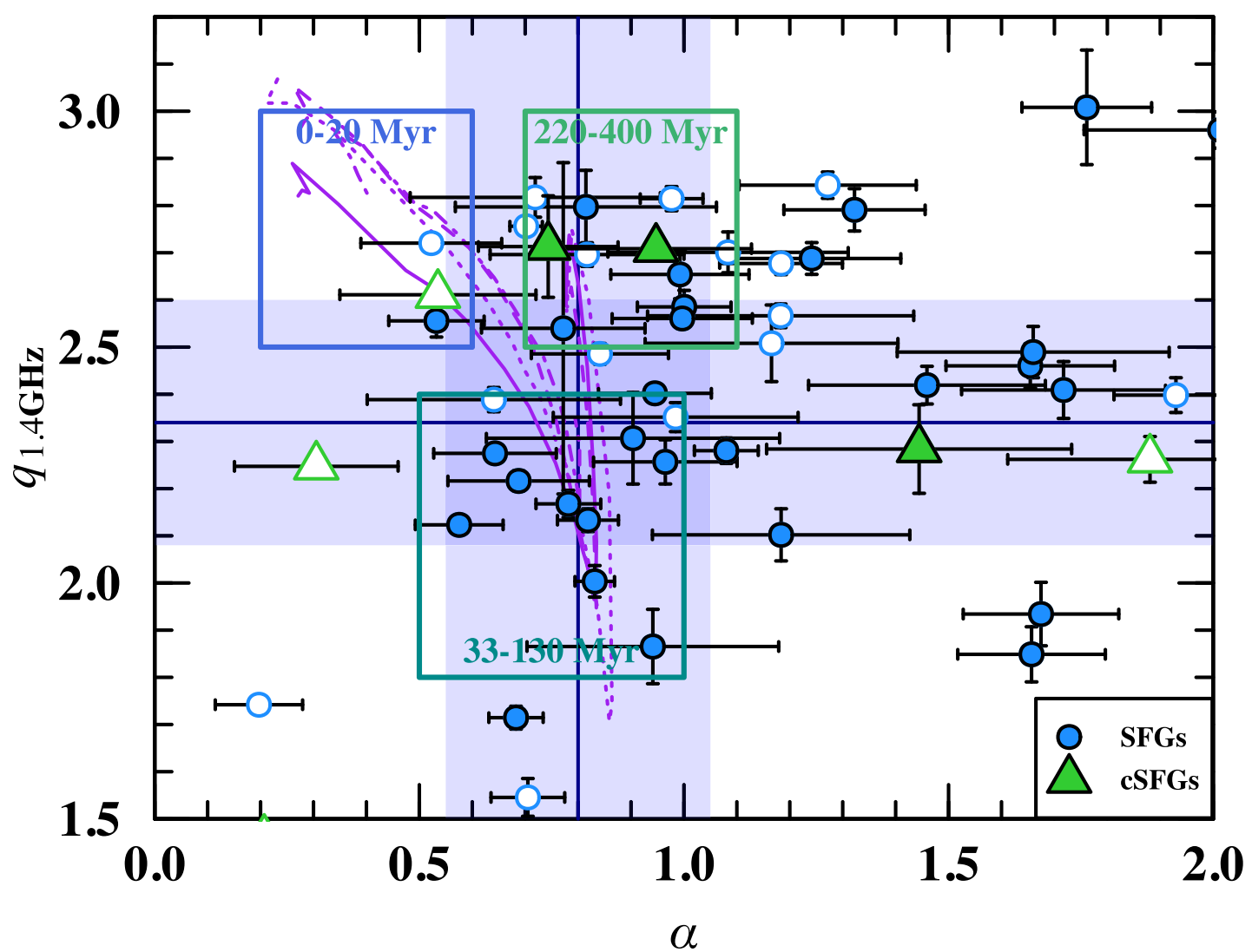

Figure 5. $q_{1.4 \mathrm{GHz}}-\alpha$ plane. The $1 \sigma$ scatter of typical values of $q_{1.4 \mathrm{GHz}}(0.26 \mathrm{dex}$; Yun et al. 2001$)$ and $\alpha(\sim 0.25 \mathrm{dex})$ for normal SFGs are represented as blue shaded regions. The evolutionary tracks for SBs of Bressan et al. (2002) are plotted as purple lines (different linetypes are examples of different $t_{\mathrm{burst}}$ in the models). The

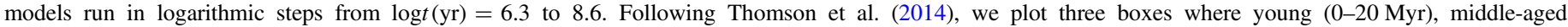
(33-130 Myr), and old (220-400 Myr) SBs are expected to be located. AGN-flagged galaxies are represented with empty symbols. Note that some AGN-flagged sources fall outside the plotted region, but we zoom in to the area of interest.

Ibar et al. 2009, 2010) and $q_{1.4 \mathrm{GHz}}=2.34 \pm 0.26$ in the local universe (e.g., Yun et al. 2001).

We calculated $\alpha$ and $q_{1.4 \mathrm{GHz}}$ for the galaxies in the radio subset of the FIR sample. $\alpha$ was obtained through fitting a single power law to the data $\left(\chi^{2}\right.$ minimization). This corresponds to the slope in the ranges $325 \mathrm{MHz}-3 \mathrm{GHz}$ for galaxies in COSMOS and $610 \mathrm{MHz}-1.4 \mathrm{GHz}$ for galaxies in GOODS-North. The FIR/radio ratio at $1.4 \mathrm{GHz}$ is defined as

$$
q_{1.4 \mathrm{GHz}}=\log \frac{L_{\mathrm{IR}}(\mathrm{W})}{3.75 \times 10^{12}(\mathrm{~Hz})}-\log \left(L_{1.4 \mathrm{GHz}}\left(\mathrm{W} \mathrm{Hz}^{-1}\right)\right)
$$

(e.g., Helou et al. 1985; Yun et al. 2001; Magnelli et al. 2015), where $L_{1.4 \mathrm{GHz}}$ was calculated via the single power law fit to the data. The uncertainties were obtained over 10,000 realizations of the observed radio SED, perturbing the photometry within the errors. In Figure 5 we present the locus of our radio subset of the FIR sample in the $q_{1.4 \mathrm{GHz}}-\alpha$ plane along with the evolutionary tracks of SBs from Bressan et al. (2002).

The distribution of galaxies is scattered around the values for normal SFGs with some outliers. Among the outliers we found ultra-steep spectrum (USS) galaxies $(\alpha>1)$, typically with low $L_{\text {radio. }}$ A similar distribution of galaxies was found in Thomson et al. (2014). The nature of USS galaxies is debated and beyond the scope of our work. Early-stage mergers are capable of steepening the radio spectrum and enhancing the radio emission (Murphy 2013). Thomson et al. (2014) also argued that an alternative scenario for USS galaxies is that they have radio jet emission truncated by interactions with dense gas in their environments (O'Dea 1998).

The location of cSFGs seems to be slightly biased toward the tracks of Bressan et al. (2002) at older ages (220-400 Myr). We checked whether there exists a trend in $\Delta \Sigma_{\mathrm{QGs}}$, and thus in compactness, along these tracks. Following Thomson et al. (2014), we divide the parameter space overlapping the tracks of Bressan et al. (2002) into three boxes representing young (0-20 Myr), middle-aged (33-130 Myr), and old (220-400 Myr) SBs. We considered all extended SFGs and cSFGs, removing the galaxies classified as AGNs because the tracks of Bressan et al. (2002) refer only to pure star formation and the contribution from the AGNs to the radio spectrum could bias the interpretation. We found that $\Delta \Sigma_{\mathrm{QGs}}$ grows with the age of the starburst episode (see Table 2), from $\Delta \Sigma_{\mathrm{QGs}}=0.137$ to $\Delta \Sigma_{\mathrm{QGs}}=0.55 \pm 0.63$. These values correspond to the median; the uncertainty is given by the median absolute deviation (MAD). Note that since the increase in compactness is a continuous function, we expect the scatter of each bin given by the MAD to overlap (as in Figure 1). We did not find a similar trend in the case of the stellar mass to that reported in Thomson et al. (2014), although to make a proper comparison selection effects should be considered.

Overall, we find a trend of increasing compactness with the evolution of a starburst episode, leading to cSFGs in the final stages. This indicates that cSFGs could be old SBs, while extended SFGs could be a mix of normal SFGs and young SBs. 
Table 2

Properties of Galaxies in Different Age Bins

\begin{tabular}{lccc}
\hline \hline $\begin{array}{l}\text { Age } \\
(\mathrm{Myr})\end{array}$ & $\log \left(M_{*} / M_{\odot}\right)$ & $\Delta \mathrm{MS}$ & $\Delta \Sigma_{\mathrm{QGs}}$ \\
\hline $0-20$ & 10.67 & 6.44 & 0.137 \\
$33-130$ & $10.62 \pm 0.37$ & $3.5 \pm 1.1$ & $0.21 \pm 0.20$ \\
$220-400$ & $10.66 \pm 0.19$ & $5.14 \pm 0.69$ & $0.55 \pm 0.63$ \\
\hline
\end{tabular}

Note. The uncertainties refer to the MAD of the galaxies in each bin, and thus no dispersion is shown in the young (0-20 Myr) age bin since there is just one galaxy. AGNs are excluded.

\section{Discussion \\ 5.1. Compactness, Star Formation, and the Diagnostics of Burstiness}

In Section 3 we presented the distribution of the general galaxy population in the $\Delta \mathrm{MS}-\Delta \Sigma_{\mathrm{QGs}}$ plane. The general evolutionary trend of the galaxy population is given by the L-shape, previously reported in Barro et al. (2017a). Extended SFGs become compact before they quench (see Figure 6 for a schematic plot). We stress that SFGs do not evolve into QGs at the same epoch (i.e., redshift), but into QGs at later times.

Furthermore, we showed two additional behaviors of the general evolutionary trend of the galaxy population: (1) galaxies start to transition smoothly toward quiescence, as the median sSFR decreases continuously for increasing compactness; (2) at least some of the SFGs become compact by increasing their sSFR and going above the scatter of the MS, given the large scatter and upper extreme values around the $\Delta \Sigma_{\mathrm{QGs}} \sim 1.0$ threshold for compactness transition.

In Section 4 we examined three indicators of the burstiness of star formation: (1) SFE, (2) ISM, and (3) radio emission.

Regarding SFE, there is no difference between cSFGs and extended SFGs. The similar values for cSFGs and extended SFGs extend to various regimes. There are both cSFGs and extended SFGs with low SFE due to high gas fractions, and also both cSFGs and extended SFGs with enhanced SFE, some of which have enhanced SFE due to low gas fractions (see Figure 3). All together, this suggests that compactness could arise from different origins, such as an extended normal SFG with low efficiency and a large gas reservoir that is secularly growing its stellar core, or an extended SB with enhanced efficiency that is rapidly consuming its gas reservoir and growing its stellar core.

In terms of the ISM, the mild excitation and density values for extended SFGs in the upper MS (see Figure 4 and Table 1) are in line with the scenario of Tacchella et al. (2016), which related the scatter of the MS to the evolution of galaxies following compaction events as part of the secular evolution of SFGs (e.g., Dekel et al. 2013; Zolotov et al. 2015; Tacchella et al. 2016). If it arises as the product of a rapid starburst-like event the latent ISM has similar properties to those of upperMS normal SFGs.

On the other hand, regarding the radio emission diagnostic, the increasing compactness with the expected evolution of the radio emission in SBs leads to the conclusion that cSFGs could be old SBs. Note also that most of the galaxies are above the scatter of the MS in this part of the analysis, particularly in the old age bin. This suggests that the galaxies displaying high a sSFR that goes above the scatter of the MS when they become cSFGs (see Figure 1) are old SBs.

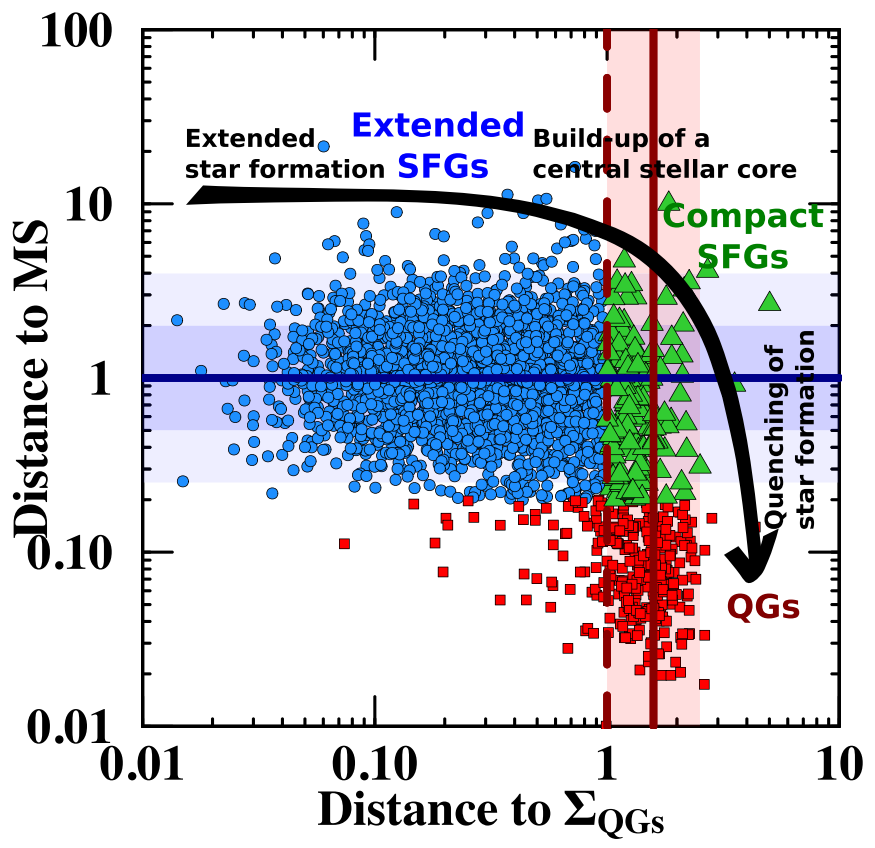

Figure 6. Outline of the general evolutionary trend of the galaxy population.

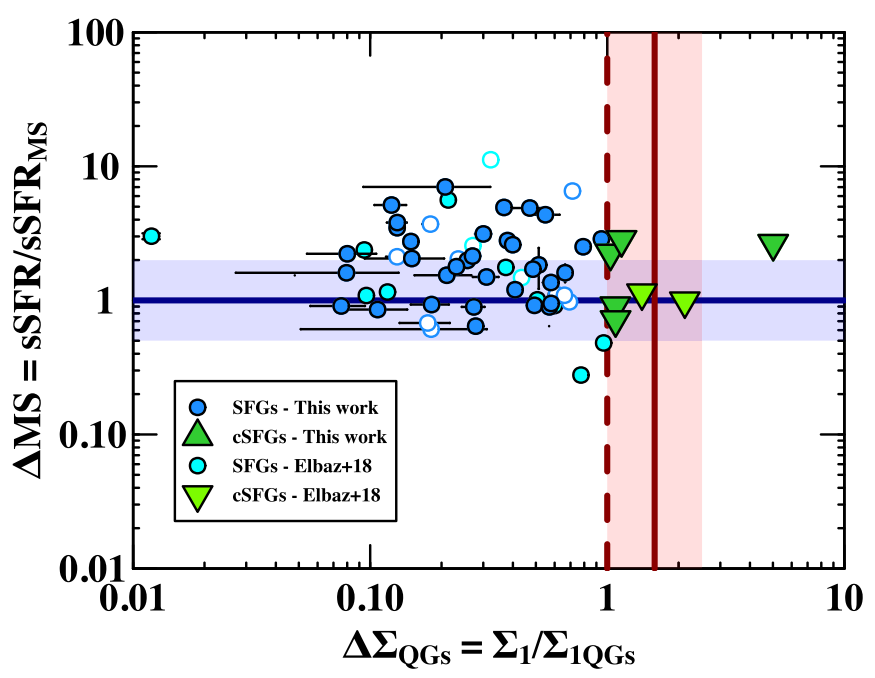

Figure 7. $\Delta \mathrm{MS}-\Delta \Sigma_{\mathrm{QGs}}$ plane as presented in Figure 1 for the R-J subset of the FIR sample and the sample of Elbaz et al. (2018). The $\Delta \Sigma_{\mathrm{QGs}}$ uncertainties were calculated over 1000 realizations, varying the structural parameters within their uncertainties (note that in some cases the uncertainties are smaller than the symbol size).

The analysis carried out in our work was performed in an unresolved fashion. This could be the reason for the apparent contradictory conclusions drawn from the SFE and ISM diagnostics versus the radio emission diagnostic. The conclusions drawn from the former can be reconciled with those from the latter if the SFE and ISM properties do not dominate the entire galaxy in an old SB phase. In that case the galaxy would not display an overall (unresolved) high SFE or SB-like ISM (see Renaud et al. 2019). This scenario would also be supported by the handful of resolved follow-up studies of the ISM of cSFGs, which indicate an ongoing nuclear starburst (e.g., Spilker et al. 2016; Barro et al. 2017b; Popping et al. 2017; Tadaki et al. 2017; Talia et al. 2018). 

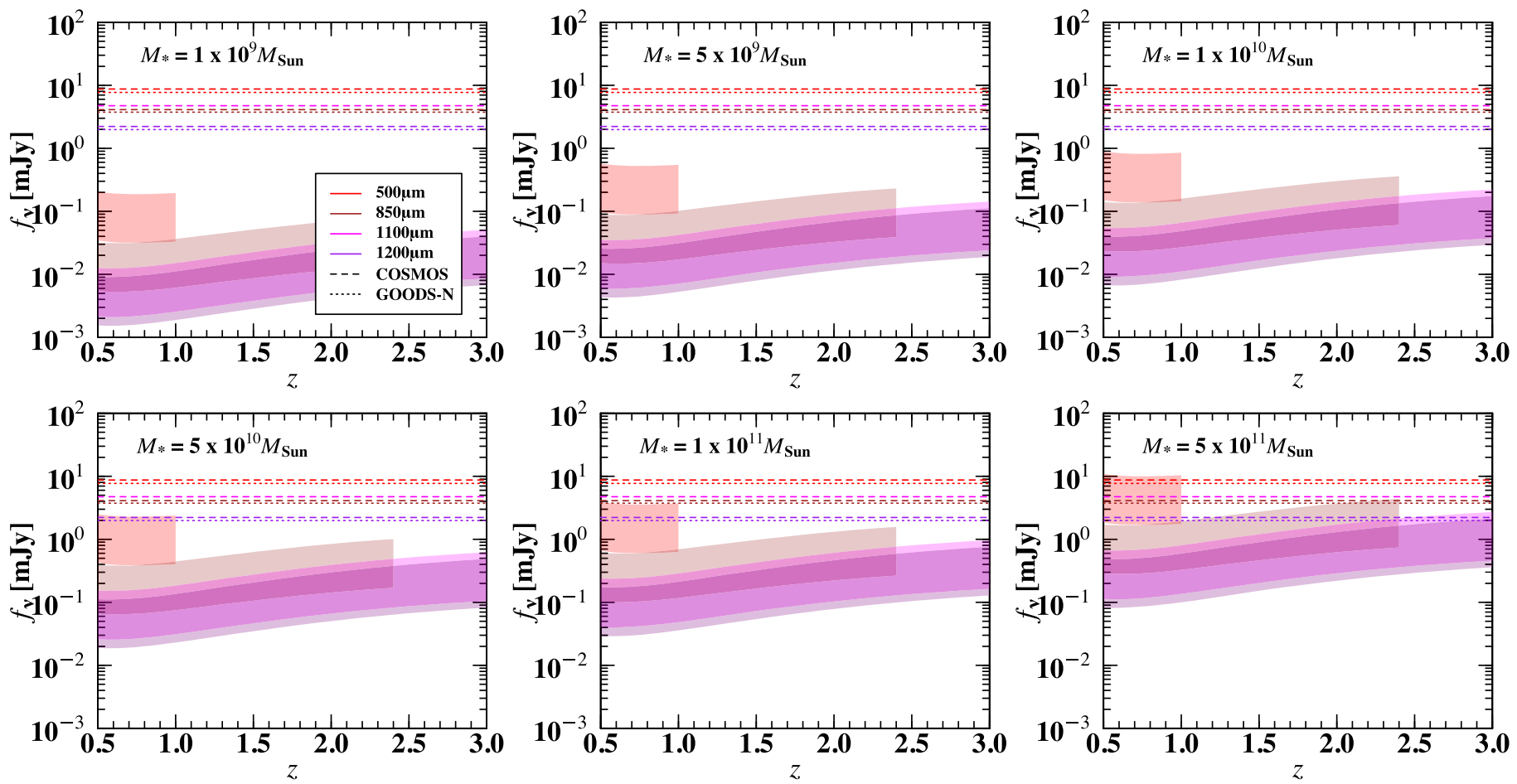

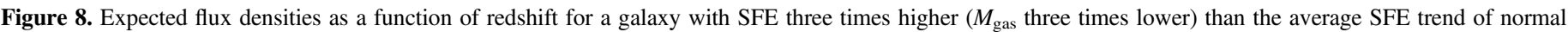

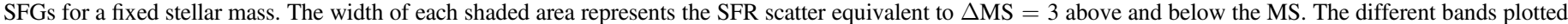

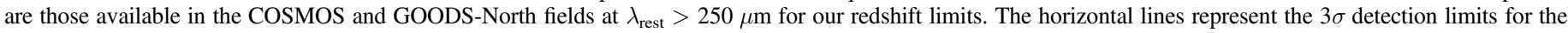

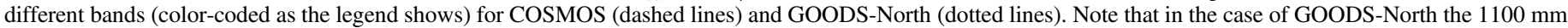
and $1200 \mathrm{~mm}$ bands are combined, and thus the detection limit refers to $1160 \mathrm{~mm}$.

\subsection{SFE and Selection Limits}

We indicated in Section 4.1 that the combination of our sample with that of Elbaz et al. (2018) implied no difference between cSFGs and extended SFGs in terms of their SFE. However, it could be the case that the galaxies of Elbaz et al. (2018) are closer to the $\Delta \Sigma_{\mathrm{QGs}} \sim 1.0$ transition threshold, particularly for the subset with high SFE and low $f_{\text {gas }}$ that could be in the last stage before becoming quiescent. In Figure 7 we show the location of both samples in the $\Delta \mathrm{MS}-\Delta \Sigma_{\mathrm{QGs}}$ plane in order to explore this scenario. We did not find evidence that that the latter is the case because we do not see any distinction between our sample and that of Elbaz et al. (2018) in the $\Delta \mathrm{MS}-\Delta \Sigma_{\mathrm{QGs}}$ plane.

In Section 4.1 we also mentioned that our sample and that of Elbaz et al. (2018) are located in distinct complementary regions in the different diagrams shown in Figure 3. For instance, we did not find galaxies with simultaneously high SFE and low $f_{\text {gas }}$ within our sample, the galaxies described in Elbaz et al. (2018) as SBs that also fall within the scatter of the MS. The galaxies of Elbaz et al. (2018) occupy a parameter space offset from our sample, which made us consider the possibility that our selection is biased against the detection of SBs within the MS.

The R-J subset of the FIR sample was selected to fulfill a detection criterion on the R-J side of the FIR SED, thereby establishing a detection limit for the different bands available on the R-J side of the "super-deblended" FIR catalogs in COSMOS and GOODS-North. For galaxies at $0.5<z<3.0$ these bands are Herschel/SPIRE $500 \mu \mathrm{m}$, SCUBA $850 \mu \mathrm{m}$, AzTEC $1.1 \mathrm{~mm}$, and MAMBO $1.2 \mathrm{~mm}$. We explored the required fluxes in these bands as a function of redshift to be able to detect galaxies that, while located within the scatter of the MS, exhibited enhanced SFE. We employed the technique of Scoville et al. (2016) to predict the single-band flux measurement of the dust continuum expected for a given $M_{\text {gas }}$. In Figure 8 we plot the predicted fluxes for the different bands as a function of redshift for galaxies with an SFE three times higher $\left(M_{\text {gas }}\right.$ three times lower) than the SFE trend of normal SFGs at fixed stellar mass for a range of SFRs equivalent to $\Delta \mathrm{MS}=3$ both above and below the MS. Only galaxies with $M_{*} \geqslant 5 \times 10^{11} M_{\odot}$ start to be detectable. These detection limits are consistent with the fact that we did not detect galaxies with enhanced SFE within the scatter of the MS. In Figure 3 we show as a reference the most favorable $f_{\text {gas }}$ limit (gray shaded region), which would correspond to a galaxy that has a stellar mass as high as the highest stellar mass of the sample $\left(\log \left(M_{*} / M_{\odot}\right)=11.73\right)$ and is located at a redshift such that the predicted detectable $f_{\text {gas }}$ given the flux limits in the different bands in the two fields results in a minimum. Even in this extreme case, the fact that there is no galaxy in our sample that fulfills all the criteria at the same time suggests that we missed galaxies with simultaneously high SFE and low $f_{\text {gas }}$, such as those presented in Elbaz et al. (2018).

Therefore, we did not find a SB-like SFE within the MS due to the detection limits in the catalogs.

\section{Summary and Conclusions}

In this work we studied the general population of galaxies based on their location with respect to fundamental starforming and structural relations, and classified them into extended SFGs, compact SFGs, and QGs. Based on a methodology of three diagnostics of the burstiness of star formation-(1) SFE, (2) ISM, and (3) radio emission-we aimed at studying whether cSFGs can be considered as normal 
SFGs or SBs. Unveiling the nature of cSFGs as a proposed immediate transition population toward QGs implies understanding how the build-up of compact stellar cores and subsequent quenching of star formation happen. If cSFGs were normal SFGs it would point toward a secular transition toward quiescence; conversely, an SB nature of cSFGs would point toward a more rapid transition toward quiescence. In summary we found:

1. The distribution of galaxies in the $\Delta \mathrm{MS}-\Delta \Sigma_{\mathrm{QGs}}$ plane reveals that galaxies transition smoothly toward quiescence with increasing compactness. Some extended SFGs quench, forming extended QGs. Most of the extended SFGs become compact before they quench, in agreement with Barro et al. (2017a). Furthermore, at least some galaxies become compact going above the scatter of the MS.

2. The MS is dominated by extended SFGs. However, SFGs with increasing compactness that are transitioning to quiescence contribute to lowering the normalization of the MS.

3. There is no evidence for a distinct SFE in cSFGs and extended SFGs, suggesting that both secular and rapid evolution processes could generate cSFGs.

4. Extended SFGs located slightly above the MS (upper-MS galaxies) have ISM properties (CO excitation, density of the neutral gas, and strength of the ultraviolet) similar to the lower envelope of SB-like ISM properties, and seem also similar to those of cSFGs (with the caveat of the small sample sizes and the lack of ISM characterization for cSFGs). This suggests that the growth of a compact stellar core leading to a cSFG could happen secularly. Another explanation could be that, if coming from a rapid starburst event, the latent ISM in cSFGs retains similar properties to that of upper-MS normal SFGs.

5. There is evidence for a trend in increasing compactness with the expected evolution of the radio emission in SBs, indicating that cSFGs could be old SBs, while extended SFGs could be a mix of normal SFGs and young SBs.

The relative importance of a secular or rapid transition toward quiescence as a function of redshift remains to be completely understood. The apparent contradictory conclusions drawn from the SFE and ISM diagnostics versus the radio emission diagnostic can be reconciled if the SFE and ISM properties do not dominate the entire galaxy in an old SB phase, in agreement with resolved follow-up studies in the literature. We suggest that cSFGs could be SBs winding down and eventually crossing the main sequence toward quiescence.

We are very grateful to D. Elbaz, N. M. Förster Schreiber, and D. Watson for the assessment of this work. We thank A. Bressan and A. P. Thomson for their support on the starburst evolutionary tracks in radio. We are grateful to the anonymous referee, whose comments have been very useful to improving our work.

C.G.G. and S.T. acknowledge support from the European Research Council (ERC) Consolidator Grant funding scheme (project ConTExt, grant number: 648179). G.E.M. and F.V. acknowledge the Villum Fonden research grant 13160 "Gas to stars, stars to dust: tracing star formation across cosmic time." A.M. is supported by the Dunlap Fellowship through an endowment established by the David Dunlap family and the University of Toronto.

The Cosmic Dawn Center is funded by the Danish National Research Foundation.

\section{ORCID iDs}

C. Gómez-Guijarro (1) https://orcid.org/0000-0002-4085-9165

G. E. Magdis (ib https://orcid.org/0000-0002-4872-2294

F. Valentino (1) https://orcid.org/0000-0001-6477-4011

S. Toft (1) https://orcid.org/0000-0003-3631-7176

R. J. Ivison (1) https://orcid.org/0000-0001-5118-1313

G. Brammer (1) https://orcid.org/0000-0003-2680-005X

\section{References}

Alaghband-Zadeh, S., Chapman, S. C., Swinbank, A. M., et al. 2013, MNRAS, 435,1493

Avni, Y. 1976, ApJ, 210, 642

Barro, G., Faber, S. M., Koo, D. C., et al. 2017a, ApJ, 840, 47

Barro, G., Faber, S. M., Pérez-González, P. G., et al. 2013, ApJ, 765, 104

Barro, G., Faber, S. M., Pérez-González, P. G., et al. 2014, ApJ, 791, 52

Barro, G., Kriek, M., Pérez-González, P. G., et al. 2017b, ApJL, 851, L40

Berta, S., Lutz, D., Genzel, R., Förster-Schreiber, N. M., \& Tacconi, L. J. 2016, A\&A, 587, A73

Bothwell, M. S., Smail, I., Chapman, S. C., et al. 2013, MNRAS, 429, 3047 Brammer, G. B., van Dokkum, P. G., Franx, M., et al. 2012, ApJS, 200, 13 Bressan, A., Silva, L., \& Granato, G. L. 2002, A\&A, 392, 377

Brinchmann, J., Charlot, S., White, S. D. M., et al. 2004, MNRAS, 351, 1151

Chabrier, G. 2003, PASP, 115, 763

Cimatti, A., Cassata, P., Pozzetti, L., et al. 2008, A\&A, 482, 21

Civano, F., Marchesi, S., Comastri, A., et al. 2016, ApJ, 819, 62

Condon, J. J. 1992, ARA\&A, 30, 575

Daddi, E., Bournaud, F., Walter, F., et al. 2010a, ApJ, 713, 686

Daddi, E., Dannerbauer, H., Liu, D., et al. 2015, A\&A, 577, A46

Daddi, E., Dickinson, M., Morrison, G., et al. 2007, ApJ, 670, 156

Daddi, E., Elbaz, D., Walter, F., et al. 2010b, ApJL, 714, L118

Davies, R. I., Sternberg, A., Lehnert, M., \& Tacconi-Garman, L. E. 2003, ApJ, 597, 907

de Jong, T., Klein, U., Wielebinski, R., \& Wunderlich, E. 1985, A\&A, 147, L6 Dekel, A., Zolotov, A., Tweed, D., et al. 2013, MNRAS, 435, 999

Del Moro, A., Alexander, D. M., Mullaney, J. R., et al. 2013, A\&A, 549, A59 Di Matteo, P., Bournaud, F., Martig, M., et al. 2008, A\&A, 492, 31

Dickinson, M., Giavalisco, M. \& GOODS Team 2003, in The Mass of Galaxies at Low and High Redshift, ed. R. Bender \& A. Renzini (Berlin: Springer), 324

Draine, B. T., \& Li, A. 2007, ApJ, 657, 810

Elbaz, D., Daddi, E., Le Borgne, D., et al. 2007, A\&A, 468, 33

Elbaz, D., Leiton, R., Nagar, N., et al. 2018, A\&A, 616, A110

Feldmann, R., \& Mayer, L. 2015, MNRAS, 446, 1939

Fu, H., Cooray, A., Feruglio, C., et al. 2013, Natur, 498, 338

Genzel, R., Tacconi, L. J., Gracia-Carpio, J., et al. 2010, MNRAS, 407, 2091

Gómez-Guijarro, C., Toft, S., Karim, A., et al. 2018, ApJ, 856, 121

Grogin, N. A., Kocevski, D. D., Faber, S. M., et al. 2011, ApJS, 197, 35

Groves, B. A., Schinnerer, E., Leroy, A., et al. 2015, ApJ, 799, 96

Helou, G., Soifer, B. T., \& Rowan-Robinson, M. 1985, ApJL, 298, L7

Ibar, E., Ivison, R. J., Best, P. N., et al. 2010, MNRAS, 401, L53

Ibar, E., Ivison, R. J., Biggs, A. D., et al. 2009, MNRAS, 397, 281

Ivison, R. J., Swinbank, A. M., Smail, I., et al. 2013, ApJ, 772, 137

Jiménez-Andrade, E. F., Magnelli, B., Karim, A., et al. 2019, A\&A, 625, A114

Jin, S., Daddi, E., Liu, D., et al. 2018, ApJ, 864, 56

Kauffmann, G., Heckman, T. M., Tremonti, C., et al. 2003, MNRAS, 346, 1055

Kaufman, M. J., Wolfire, M. G., \& Hollenbach, D. J. 2006, ApJ, 644, 283

Kaufman, M. J., Wolfire, M. G., Hollenbach, D. J., \& Luhman, M. L. 1999, ApJ, 527, 795

Kennicutt, R. C., Jr. 1998, ARA\&A, 36, 189

Koekemoer, A. M., Faber, S. M., Ferguson, H. C., et al. 2011, ApJS, 197, 36

Lang, P., Wuyts, S., Somerville, R. S., et al. 2014, ApJ, 788, 11

Liu, D., Daddi, E., Dickinson, M., et al. 2018, ApJ, 853, 172

Magdis, G. E., Daddi, E., Béthermin, M., et al. 2012, ApJ, 760, 6

Magdis, G. E., Rigopoulou, D., Daddi, E., et al. 2017, A\&A, 603, A93

Magnelli, B., Ivison, R. J., Lutz, D., et al. 2015, A\&A, 573, A45

Malhotra, S., Kaufman, M. J., Hollenbach, D., et al. 2001, ApJ, 561, 766 
Marchesi, S., Civano, F., Elvis, M., et al. 2016, ApJ, 817, 34

Momcheva, I. G., Brammer, G. B., van Dokkum, P. G., et al. 2016, ApJS, 225,27

Murphy, E. J. 2013, ApJ, 777, 58

Nelson, E., van Dokkum, P., Franx, M., et al. 2014, Natur, 513, 394

Noeske, K. G., Weiner, B. J., Faber, S. M., et al. 2007, ApJL, 660, L43

O’Dea, C. P. 1998, PASP, 110, 493

Pettini, M., \& Pagel, B. E. J. 2004, MNRAS, 348, L59

Popping, G., Decarli, R., Man, A. W. S., et al. 2017, A\&A, 602, A11

Pound, M. W., \& Wolfire, M. G. 2008, in ASP Conf. Ser. 394, Astronomical

Data Analysis Software and Systems XVII, ed. R. W. Argyle,

P. S. Bunclark, \& J. R. Lewis (San Francisco, CA: ASP), 654

Puglisi, A., Daddi, E., Liu, D., et al. 2019, ApJL, 877, L23

Renaud, F., Bournaud, F., Agertz, O., et al. 2019, A\&A, 625, A65

Ricciardelli, E., Trujillo, I., Buitrago, F., \& Conselice, C. J. 2010, MNRAS, 406, 230

Rodighiero, G., Brusa, M., Daddi, E., et al. 2015, ApJL, 800, L10

Sargent, M. T., Daddi, E., Béthermin, M., et al. 2014, ApJ, 793, 19

Schawinski, K., Urry, C. M., Virani, S., et al. 2010, ApJ, 711, 284

Schinnerer, E., Groves, B., Sargent, M. T., et al. 2016, ApJ, 833, 112

Schmidt, M. 1959, ApJ, 129, 243

Schreiber, C., Pannella, M., Elbaz, D., et al. 2015, A\&A, 575, A74

Scoville, N., Aussel, H., Brusa, M., et al. 2007, ApJS, 172, 1

Scoville, N., Aussel, H., Sheth, K., et al. 2014, ApJ, 783, 84

Scoville, N., Lee, N., Vanden Bout, P., et al. 2017, ApJ, 837, 150

Scoville, N., Sheth, K., Aussel, H., et al. 2016, ApJ, 820, 83

Skelton, R. E., Whitaker, K. E., Momcheva, I. G., et al. 2014, ApJS, 214, 24
Speagle, J. S., Steinhardt, C. L., Capak, P. L., \& Silverman, J. D. 2014, ApJS, 214, 15

Spilker, J. S., Bezanson, R., Marrone, D. P., et al. 2016, ApJ, 832, 19

Tacchella, S., Dekel, A., Carollo, C. M., et al. 2016, MNRAS, 457, 2790

Tacconi, L. J., Genzel, R., Neri, R., et al. 2010, Natur, 463, 781

Tacconi, L. J., Genzel, R., Saintonge, A., et al. 2018, ApJ, 853, 179

Tacconi, L. J., Genzel, R., Smail, I., et al. 2008, ApJ, 680, 246

Tadaki, K.-I., Kodama, T., Nelson, E. J., et al. 2017, ApJL, 841, L25

Tal, T., Dekel, A., Oesch, P., et al. 2014, ApJ, 789, 164

Talia, M., Pozzi, F., Vallini, L., et al. 2018, MNRAS, 476, 3956

Thomson, A. P., Ivison, R. J., Simpson, J. M., et al. 2014, MNRAS, 442, 577

Tisanić, K., Smolčić, V., Delhaize, J., et al. 2019, A\&A, 621, A139

Toft, S., Smolčić, V., Magnelli, B., et al. 2014, ApJ, 782, 68

Toft, S., Zabl, J., Richard, J., et al. 2017, Natur, 546, 510

Valentino, F., Magdis, G. E., Daddi, E., et al. 2018, ApJ, 869, 27

van der Wel, A., Bell, E. F., Häussler, B., et al. 2012, ApJS, 203, 24

van der Wel, A., Franx, M., van Dokkum, P. G., et al. 2014, ApJ, 788, 28

van Dokkum, P. G., Bezanson, R., van der Wel, A., et al. 2014, ApJ, 791, 45

van Dokkum, P. G., Nelson, E. J., Franx, M., et al. 2015, ApJ, 813, 23

Whitaker, K. E., Bezanson, R., van Dokkum, P. G., et al. 2017, ApJ, 838, 19

Whitaker, K. E., Franx, M., Leja, J., et al. 2014, ApJ, 795, 104

Whitaker, K. E., van Dokkum, P. G., Brammer, G., \& Franx, M. 2012, ApJL, 754, L29

Williams, C. C., Giavalisco, M., Cassata, P., et al. 2014, ApJ, 780, 1

Xue, Y. Q., Luo, B., Brandt, W. N., et al. 2016, ApJS, 224, 15

Yun, M. S., Reddy, N. A., \& Condon, J. J. 2001, ApJ, 554, 803

Zolotov, A., Dekel, A., Mandelker, N., et al. 2015, MNRAS, 450, 2327 